\title{
Categorical Abstract Algebraic Logic: Truth-Equational $\pi$-Institutions
}

\author{
George Voutsadakis
}

\begin{abstract}
Finitely algebraizable deductive systems were introduced by Blok and Pigozzi to capture the essential properties of those deductive systems that are very tightly connected to quasivarieties of universal algebras. They include the equivalential logics of Czelakowski. Based on Blok and Pigozzi's work, Herrmann defined algebraizable deductive systems. These are the equivalential deductive systems that are also truth-equational, in the sense that the truth predicate of the class of their reduced matrix models is explicitly definable by some set of unary equations. Raftery undertook the task of characterizing the property of truth-equationality for arbitrary deductive systems. In this paper, following Raftery, we extend the notion of truth-equationality for logics formalized as $\pi$-institutions and abstract several of the results that hold for deductive systems in this more general categorical context.
\end{abstract}

\section{Introduction}

A deductive system $\delta=\left\langle\mathscr{L}, \vdash_{s}\right\rangle$ consists of a logical language $\mathscr{L}=\langle\Lambda, \rho\rangle$, that is, a set of connectives $\Lambda$, each of finite arity given by the arity function $\rho: \Lambda \rightarrow \omega$, and a structural consequence relation $\vdash_{\delta} \subseteq \mathcal{P}\left(\mathrm{Fm}_{\mathscr{L}}(V)\right) \times \mathrm{Fm}_{\mathscr{L}}(V)$ on the set of formulas $\operatorname{Fm}_{\mathscr{L}}(V)$, formed using the connectives in $\mathscr{L}$ and variables in a fixed denumerable set $V$ in the ordinary recursive way. An $\mathscr{L}$-matrix $\mathfrak{A}=\langle\mathbf{A}, F\rangle$ is a pair consisting of an $\mathscr{L}$-algebra $\mathbf{A}=\left\langle A, \mathscr{L}^{\mathbf{A}}\right\rangle$ together with a subset $F \subseteq A$ of its carrier $A$. The $\mathscr{L}$-matrix $\mathfrak{A}$ is called a matrix model of $\delta$ or an $\delta$-matrix if $F$ is an $\delta$-filter, that is, if it is closed under all derivable rules of $\delta$ meaning that, for all $\Phi \cup\{\varphi\} \subseteq \operatorname{Fm}_{\mathscr{L}}(V)$, such that $\Phi \vdash_{\&} \varphi$, and every homomorphism $h: \mathbf{F m}_{\mathscr{L}}(V) \rightarrow \mathbf{A}$ from the absolutely free $\mathscr{L}$-algebra to the underlying algebra $\mathbf{A}$ of $\mathfrak{U}$,

$$
h(\Phi) \subseteq F \quad \text { implies } \quad h(\varphi) \in F .
$$

Received June 27, 2011; accepted April 20, 2013

2010 Mathematics Subject Classification: Primary 03G27

Keywords: deductive system, logical matrix, Leibniz congruence, Suszko congruence, truth-equational logics, algebraizable logics

(C) 2015 by University of Notre Dame 10.1215/00294527-2864343 
The collection of all $\delta$-filters on $\mathbf{A}$ is denoted by $\mathrm{Fi}_{\&} \mathbf{A}$. Notice that, taking into account structurality, the $\delta$-filters on the formula algebra correspond exactly to the theories of the deductive system $\delta$, that is, the sets of formulas that are closed under the entailment $\vdash_{8}$. Given an $\mathscr{L}$-matrix $\mathfrak{U}=\langle\mathbf{A}, F\rangle$, there always exists a largest congruence on $\mathbf{A}$ that is compatible with $F$. Compatibility of a congruence $\theta$ with $F$ means that, for all $a, b \in A$, if $\langle a, b\rangle \in \theta$ and $a \in F$, then $b \in F$ or, equivalently, that $F$ is a union of $\theta$-equivalence classes. This largest congruence is called the Leibniz congruence of $F$ and denoted by $\Omega_{\mathbf{A}}(F)$ or $\Omega(\mathfrak{U})$ (see Blok and Pigozzi [3]). When $\Omega$ refers to matrices on the formula algebra, the subscript referring to the formula algebra $\mathbf{F m}_{\mathscr{L}}(V)$ is usually omitted. On the other hand, the largest congruence on $\mathbf{A}$ that is compatible with all $\&$-filters on $\mathbf{A}$ including $F$, which also always exists, is termed the Suszko congruence on $\mathbf{A}$ and is denoted by $\tilde{\Omega}_{\mathbf{A}}(F)$ (see Czelakowski [9]). Let $\delta=\left\langle\mathscr{L}, \vdash_{\S}\right\rangle$ be a deductive system, and let $\mathfrak{U}=\langle\mathbf{A}, F\rangle$ be an $\mathscr{L}$-matrix. The matrix $\mathfrak{U}$ will be said to be Leibniz reduced if $\Omega_{\mathbf{A}}(F)$ is the identity relation on $\mathbf{A}$. It is called Suszko reduced if $\tilde{\Omega}_{\mathbf{A}}(F)$ is the identity relation on $\mathbf{A}$. The collection of all $\delta$-matrices is denoted by Mod $\delta$, the collection of all Leibniz reduced $\delta$-matrices by $\operatorname{Mod}^{*} \delta$, and the collection of all Suszko reduced $\delta$-matrices by $\operatorname{Mod}^{\mathrm{Su}} \delta$.

The Leibniz operator, seen as a function $\Omega: \operatorname{Th} \delta \rightarrow \operatorname{Con}\left(\mathbf{F m}_{\mathscr{L}}(V)\right)$ from the collection of theories of $\delta$ to the collection of all $\mathscr{L}$-congruences on the formula algebra, is the function that assigns to every theory $T$ of $\delta$ its Leibniz congruence $\Omega(T)$. Properties of this operator have played a crucial role in classifying deductive systems into an algebraic hierarchy reflecting the nature of their algebraic character. This classification process, along with studies relating to the algebraic counterparts of deductive systems, constitutes the heart of the field of abstract algebraic logic (see Czelakowski [6]-[8], Blok and Pigozzi [2], [3], Font and Jansana [11], Font, Jansana, and Pigozzi [12]).

In their seminal Memoirs monograph [3], Blok and Pigozzi defined algebraizable deductive systems. The definition pertained to finitary deductive systems. Roughly speaking, a finitary deductive system is called algebraizable if there exist finitary interpretations between its consequence relation and the equational consequence relation associated with a class of algebras that are inverse of one another in a precise technical sense. Herrmann [18], [19] extended this definition to possibly infinitary deductive systems by allowing also infinitary interpretations. Herrmann's notion became known as algebraizability, whereas the original notion of Blok and Pigozzi is now known as finite algebraizability. Czelakowski, in another important work in the field of abstract algebraic logic, had previously studied equivalential logics (see [6], [7]). These were defined by Prucnal and Wronski [22] and are, again roughly speaking, those logics for which there exists a translation from the equational consequence of a class of algebras into their own consequence relation. One of the adages put forward by Herrmann in his Ph.D. dissertation [20] was that

$$
\text { Algebraizability }=\text { Truth-Equationality }+ \text { Equivalentiality. }
$$

In fact, truth-equationality is the property that fills in the interpretation from the consequence relation of the deductive system under consideration into the equational consequence of a class of algebras in order to establish algebraizability starting from equivalentiality. Until 2006, this property had only been studied in the context of protoalgebraic logics, the wider class of logics considered amenable to algebraic 
methods and techniques (see [3], [12]). Raftery, however, studied in [23] the property of truth-equationality in the more general context of arbitrary deductive systems.

Let $\delta=\left\langle\mathscr{L}, \vdash_{s}\right\rangle$ be a deductive system. According to Raftery [23], the filters of the Leibniz reduced matrices in $\operatorname{Mod}^{*} \delta$ are equationally definable if there exists a set $\boldsymbol{\tau}$ of formal unary equations $\delta(x) \approx \epsilon(x)$, such that, for all $\mathfrak{U}=\langle\mathbf{A}, F\rangle \in \operatorname{Mod}^{*} \delta$, and all $a \in A$,

$$
a \in F \quad \text { iff } \quad \delta^{\mathbf{A}}(a)=\epsilon^{\mathbf{A}}(a) \quad \text { for all } \delta \approx \epsilon \in \boldsymbol{\tau} .
$$

The Leibniz operator of a deductive system is said to be completely order reflecting if, for every algebra $\mathbf{A}$ of the same similarity type as $\delta$ and every collection of $\delta$-filters $\tilde{F} \cup\{G\}$ on $\mathbf{A}$,

$$
\bigcap_{F \in \mathscr{F}} \Omega_{\mathbf{A}}(F) \subseteq \Omega_{\mathbf{A}}(G) \text { implies } \bigcap \mathscr{F} \subseteq G .
$$

Furthermore, it is said to be completely order reflecting on theories if the same condition holds for arbitrary collections of theories of $\$$. In one of the main theorems of [23], Raftery shows that a deductive system is truth-equational if and only if its Leibniz operator is completely order reflecting and, moreover, that this happens if and only if the Leibniz operator is completely order reflecting on theories.

Finally, in the same work, Raftery proves that a deductive system is truthequational if and only if its Suszko operator is globally injective, that is, injective on the filters of every algebra of the similarity type of $\delta$. This result is accompanied by two negative but, nevertheless, important results: first, that injectivity of the Suszko operator on the theories of $\delta$ does not imply the truth-equationality of $\delta$ in general and, second, that the global injectivity of the Leibniz operator is not sufficient for truth-equationality either. In fact, in [23, Examples 2, 3], Raftery showcases a deductive system that has a globally injective Leibniz operator, but not only is it not truth-equational, it does not even possess an algebraic semantics (see Blok and Rebagliato [4]), a property much weaker than truth-equationality.

It is this study of Raftery that has led to the present work, where an attempt is made to lift the study of truth-equational deductive systems into the more general context of logics formalized as $\pi$-institutions. But an exposition of the main concepts introduced in the paper and an overview of the main results has to be postponed until the next section.

The paper is organized as follows. As mentioned above, in Section 2, the basic concepts on which the development of the theory is based are introduced and an overview of the main results is provided. In Section 3 we remind the reader of the definitions of the Leibniz and Suszko operators and revisit and prove several results concerning those operators that will prove useful in subsequent sections. We also take the opportunity to introduce some additional necessary notation. In Section 4, we define the key notion of truth being equationally definable by a set of equations for a given $\pi$-institution $d$. We provide characterizations based on the classes of Leibniz-reduced and Suszko-reduced matrix system models of the $\pi$-institution and their properties. Criteria that can be used to test for the equational definability of truth are detailed in Section 5. In the final section, Section 6, all previous notions are put together under the unifying central notion of a truth-equational $\pi$-institution. Besides a chain of implications that connects various previous statements concerning properties of a given $\pi$-institution and the definability of truth, we also establish 
a condition-unfortunately, rather restrictive- under which all the previously introduced notions turn out to be equivalent. Even though this condition allows room for all $\pi$-institutions arising from deductive systems and, thus, the result encompasses the corresponding theorem, Raftery [23, Theorem 28], the condition may be too strong for arbitrary $\pi$-institutions. Refining, or perhaps relaxing, this condition will be left as a goal for future work.

\section{Preliminaries and Overview}

In this section we present the basic notions that will allow us to study some of the results of Raftery [23] in the context of logical systems formalized as $\pi$-institutions. The concept of a $\pi$-institution (see Fiadeiro and Sernadas [10]; see also Goguen and Burstall [16], [17]) constitutes the basic structure that allows the formalization of logical systems that are more general than those that can be formalized using the deductive systems of universal abstract algebraic logic (see, e.g., Voutsadakis [24], [25]). Introducing the basic notation and some of the basic related concepts will also allow us to give an overview of the contents of the paper and recount their relation with the original results of [23], which inspired their development. For the basic categorical concepts and notation that will be used in this section and the remainder of the paper, the reader is encouraged to consult any of the standard references (see Barr and Wells [1], Borceux [5], Mac Lane [21]) in general category theory.

A $\pi$-institution $d=\langle$ Sign, SEN,$C\rangle$ is a triple consisting of an arbitrary category Sign, a set-valued functor SEN : Sign $\rightarrow$ Set (in this context, sometimes termed a sentence functor), and a collection $C=\left\{C_{\Sigma}\right\}_{\Sigma \in \mid \text { Sign } \mid}$ of closure operators $C_{\Sigma}: \mathcal{P}(\operatorname{SEN}(\Sigma)) \rightarrow \mathcal{P}(\operatorname{SEN}(\Sigma)), \Sigma \in|\operatorname{Sign}|$, such that, for all $\Sigma_{1}, \Sigma_{2} \in|\operatorname{Sign}|$ and all $f \in \operatorname{Sign}\left(\Sigma_{1}, \Sigma_{2}\right)$,

$$
\operatorname{SEN}(f)\left(C_{\Sigma_{1}}(\Phi)\right) \subseteq C_{\Sigma_{2}}(\operatorname{SEN}(f)(\Phi)) .
$$

The map $C_{\Sigma}: \mathcal{P}(\operatorname{SEN}(\Sigma)) \rightarrow \mathcal{P}(\operatorname{SEN}(\Sigma))$ is a closure operator if it satisfies, for all $\Phi \subseteq \Psi \subseteq \operatorname{SEN}(\Sigma)$,

- $\Phi \subseteq C_{\Sigma}(\Phi)$ (Reflexivity),

- $C_{\Sigma}(\Phi) \subseteq C_{\Sigma}(\Psi) \quad$ (Monotonicity),

- $C_{\Sigma}\left(C_{\Sigma}(\Phi)\right)=C_{\Sigma}(\Phi) \quad$ (Idempotency).

Moreover $C$ is termed a closure system on SEN if, in addition, condition (1) holds.

The structure of a $\pi$-institution abstracts that of a deductive system, which is used as the underlying structure supporting the concept of a logical system in universal abstract algebraic logic. Categorical abstract algebraic logic aspires to abstract the methods and results of the universal treatment to a wider class of logical systems and, as a result, broaden their applicability. To achieve this goal, it uses $\pi$-institutions as the underlying supporting structures representing logical systems, because $\pi$-institutions can readily accommodate logical systems with multiple signatures and quantifiers which are more difficult to deal with using deductive systems (see, e.g., the appendix in [3] and relevant discussions in both [25] and [24]).

To abstract the concept of an algebraic signature (or logical language) from the level of deductive systems to the level of $\pi$-institutions, we consider the notion of the category of natural transformations on a given functor. Let Sign be a category, and let SEN : Sign $\rightarrow$ Set be a functor. The clone of all natural transformations on SEN is defined to be the locally small category with collection of 
objects $\left\{\operatorname{SEN}^{\alpha}: \alpha\right.$ an ordinal $\}$, collection of morphisms $\tau: \mathrm{SEN}^{\alpha} \rightarrow \mathrm{SEN}^{\beta}$, and $\beta$-sequences of natural transformations $\tau_{i}: \mathrm{SEN}^{\alpha} \rightarrow \mathrm{SEN}$. Composition

$$
\left.\left.\mathrm{SEN}^{\alpha} \longrightarrow \operatorname{SEN}^{\beta} \longrightarrow \tau_{i}: i<\beta\right\rangle=\sigma_{j}: j<\gamma\right\rangle \longrightarrow \mathrm{SEN}^{\gamma}
$$

is defined by

$$
\left\langle\sigma_{j}: j<\gamma\right\rangle \circ\left\langle\tau_{i}: i<\beta\right\rangle=\left\langle\sigma_{j}\left(\left\langle\tau_{i}: i<\beta\right\rangle\right): j<\gamma\right\rangle .
$$

A subcategory $N$ of this category containing all objects of the form $\mathrm{SEN}^{k}$ for $k<\omega$, and all projection morphisms $p^{k, i}: \mathrm{SEN}^{k} \rightarrow \mathrm{SEN}, i<k, k<\omega$, with $p_{\Sigma}^{k, i}: \operatorname{SEN}(\Sigma)^{k} \rightarrow \operatorname{SEN}(\Sigma)$ given by

$$
p_{\Sigma}^{k, i}(\vec{\varphi})=\varphi_{i}, \quad \text { for all } \vec{\varphi} \in \operatorname{SEN}(\Sigma)^{k},
$$

and such that, for every family $\left\{\tau_{i}: \mathrm{SEN}^{k} \rightarrow \mathrm{SEN}: i<l\right\}$ of natural transformations in $N$, the sequence $\left\langle\tau_{i}: i<l\right\rangle: \mathrm{SEN}^{k} \rightarrow \mathrm{SEN}^{l}$ is also in $N$, is referred to as a category of natural transformations on SEN.

Since categories of natural transformations on set-valued functors are used to abstract algebraic signatures (more precisely, clones of algebraic operations generated by specific fundamental operations), the notion of a translation between functors, which will be used in lieu of algebraic homomorphisms, will naturally be assumed to respect those categories. Let Sign be a category, let SEN : Sign $\rightarrow$ Set be a functor, and let $N$ be a category of natural transformations on SEN. Consider two categories Sign', Sign" and functors $\mathrm{SEN}^{\prime}: \mathbf{S i g n}^{\prime} \rightarrow$ Set and $\mathrm{SEN}^{\prime \prime}: \mathbf{S i g n}^{\prime \prime} \rightarrow$ Set, with $N^{\prime}$ and $N^{\prime \prime}$ categories of natural transformations on $\mathrm{SEN}^{\prime}$ and $\mathrm{SEN}^{\prime \prime}$, respectively. Assume, moreover, that there exist surjective functors $F^{\prime}: N \rightarrow N^{\prime}$ and $F^{\prime \prime}: N \rightarrow N^{\prime \prime}$ that preserve projections. In this case $N^{\prime}$ and $N^{\prime \prime}$ will be said to be similar and the natural transformations $F^{\prime}(\sigma), F^{\prime \prime}(\sigma)$ in $N^{\prime}, N^{\prime \prime}$, respectively, that correspond to $\sigma$ in $N$, will be denoted by $\sigma^{\prime}$ and $\sigma^{\prime \prime}$.

Let, again, Sign, Sign' be categories, and let SEN : Sign $\rightarrow$ Set, SEN $^{\prime}:$ Sign' $^{\prime} \rightarrow$ Set be functors, with $N, N^{\prime}$ categories of natural transformations on SEN, SEN', respectively. A pair $\langle F, \alpha\rangle$ will be said to be an $\left(N, N^{\prime}\right)$-epimorphic translation if $F:$ Sign $\rightarrow$ Sign' $^{\prime}$ is a functor and $\alpha: \operatorname{SEN} \rightarrow \operatorname{SEN}^{\prime} \circ F$ is a natural transformation, such that, for all $\sigma: \operatorname{SEN}^{n} \rightarrow \operatorname{SEN}$ in $N$, all $\Sigma \in|\operatorname{Sign}|$, and all $\vec{\varphi} \in \operatorname{SEN}(\Sigma)^{n}$,

$$
\alpha_{\Sigma}\left(\sigma_{\Sigma}(\vec{\varphi})\right)=\sigma_{F(\Sigma)}^{\prime}\left(\alpha_{\Sigma}^{n}(\vec{\varphi})\right) .
$$

Given a functor SEN : Sign $\rightarrow$ Set, with a category $N$ of natural transformations on SEN, an $N$-algebraic system $\mathcal{A}^{\prime}=\left\langle\mathrm{SEN}^{\prime},\langle F, \alpha\rangle\right\rangle$ consists of a functor $\mathrm{SEN}^{\prime}:$ Sign $^{\prime} \rightarrow$ Set, with $N^{\prime}$ a category of natural transformations on $\mathrm{SEN}^{\prime}$, together with an $\left(N, N^{\prime}\right)$-epimorphic translation $\langle F, \alpha\rangle: \mathrm{SEN} \rightarrow \mathrm{SEN}^{\prime} . N$-algebraic systems have appeared many times before in the theory of categorical abstract algebraic logic in various contexts, sometimes under disguises, and have helped in creating, for example, a model theory of $\pi$-institutions in Voutsadakis [27] and a theory for institutional logics based on the Tarski operator Voutsadakis [26], paralleling the theory on sentential logics of Font and Jansana [11].

Let $d=\langle$ Sign, SEN, $C\rangle$ be a $\pi$-institution, with $N$ a category of natural transformations on SEN. Given an $N$-algebraic system $\mathcal{A}^{\prime}$, as before, there exists a minimal closure system $C^{\mathcal{A}^{\prime}}$ on $\mathrm{SEN}^{\prime}$, such that $\langle F, \alpha\rangle: \mathcal{d} \rightarrow\left\langle\mathbf{S i g n}^{\prime}, \mathrm{SEN}^{\prime}, C^{\mathcal{A}^{\prime}}\right\rangle$ is an $\left(N, N^{\prime}\right)$-logical morphism, that is, such that $\langle F, \alpha\rangle: \mathrm{SEN} \rightarrow \mathrm{SEN}^{\prime}$ is 
an $\left(N, N^{\prime}\right)$-epimorphic translation and, moreover, for all $\Sigma \in|\mathbf{S i g n}|$ and all $\Phi \cup\{\varphi\} \subseteq \operatorname{SEN}(\Sigma)$

$$
\varphi \in C_{\Sigma}(\Phi) \quad \text { implies } \quad \alpha_{\Sigma}(\varphi) \in C_{F(\Sigma)}^{\mathcal{A}^{\prime}}\left(\alpha_{\Sigma}(\Phi)\right) .
$$

The existence of such a closure system follows from the fact that the system $C^{\top}=\left\{C_{\Sigma}^{\top}\right\}_{\Sigma \in\left|\mathbf{S i g n}^{\prime}\right|}$, which is defined for all $\Sigma \in\left|\mathbf{S i g n}^{\prime}\right|$ by

$$
C_{\Sigma}^{\top}(\Phi)= \begin{cases}\emptyset & \text { if } \Phi=\emptyset \\ \operatorname{SEN}^{\prime}(\Sigma) & \text { if } \Phi \neq \varnothing\end{cases}
$$

satisfies condition (2) and that, given any collection of closure systems $\left\{C^{i}: i \in I\right\}$ on $\mathrm{SEN}^{\prime}$, the signature-wise intersection $C^{\prime}=\bigcap_{i \in I} C^{i}$ is also a closure system. The collection of the theory families of this minimal system $C^{\mathcal{A}^{\prime}}$ on $\mathrm{SEN}^{\prime}$ generated by $\mathcal{A}^{\prime}$ has been considered before in the literature of categorical abstract algebraic logic (see, e.g., Voutsadakis [30]) and has been denoted by $\mathrm{ThFam}_{\ell}^{\langle F, \alpha\rangle}\left(\mathrm{SEN}^{\prime}\right)$. Moreover, the $\pi$-institution $d^{\mathcal{A}^{\prime}}:=\left\langle\mathbf{S i g n}^{\prime}, \mathrm{SEN}^{\prime}, C^{\mathcal{A}^{\prime}}\right\rangle$ has been termed the $\langle F, \alpha\rangle$-min $\left(N, N^{\prime}\right)$-model of $d$ on $\mathrm{SEN}^{\prime}$ (see [27]). An $N$-matrix system $\mathfrak{U}^{\prime}=\left\langle\mathcal{A}^{\prime}, T^{\prime}\right\rangle=\left\langle\left\langle\mathrm{SEN}^{\prime},\langle F, \alpha\rangle\right\rangle, T^{\prime}\right\rangle$ is a pair consisting of an $N^{\prime}$-algebraic system $\mathcal{A}^{\prime}=\left\langle\mathrm{SEN}^{\prime},\langle F, \alpha\rangle\right\rangle$ together with an axiom system $T^{\prime}=\left\{T_{\Sigma}^{\prime}\right\}_{\Sigma \in\left|\mathbf{S i g n}^{\prime}\right|}$ on $\operatorname{SEN}^{\prime}$ (i.e., a collection of subsets $T_{\Sigma}^{\prime} \subseteq \operatorname{SEN}^{\prime}(\Sigma), \Sigma \in\left|\mathbf{S i g n}^{\prime}\right|$ ). An $N$-matrix system model $\left\langle\mathcal{A}^{\prime}, T^{\prime}\right\rangle=\left\langle\left\langle\mathrm{SEN}^{\prime},\langle F, \alpha\rangle\right\rangle, T^{\prime}\right\rangle$ of $d$ is an $N$-matrix system, such that $T^{\prime} \in \operatorname{ThFam}_{l}^{\langle F, \alpha\rangle}\left(\mathrm{SEN}^{\prime}\right)$.

Before continuing our exploration of the basic notions, we propose to give an alternative view of the $N$-matrix system models, which is new (to our knowledge) and proves very useful in checking that an axiom family $T^{\prime}=\left\{T_{\Sigma}^{\prime}\right\}_{\Sigma \in\left|\mathbf{S i g n}^{\prime}\right|}$ on $\mathrm{SEN}^{\prime}$ is in fact a theory family of $C^{\mathcal{A}^{\prime}}$, for some $N$-algebraic system $\mathcal{A}^{\prime}=\left\langle\mathrm{SEN}^{\prime},\langle F, \alpha\rangle\right\rangle$. For a given $N$-matrix system $\mathfrak{H}^{\prime}=\left\langle\mathcal{A}^{\prime}, T^{\prime}\right\rangle$, define $C^{\mathfrak{Q}^{\prime}}=\left\{C_{\Sigma}^{\mathfrak{Q}^{\prime}}\right\}_{\Sigma \in \mid \text { Sign }}$, by letting, for all $\Sigma \in|\operatorname{Sign}|, C_{\Sigma}^{\mathfrak{Q} \mathcal{U}^{\prime}}: \mathcal{P}(\operatorname{SEN}(\Sigma)) \rightarrow \mathcal{P}(\operatorname{SEN}(\Sigma))$ be given, for all $\Phi \cup\{\varphi\} \subseteq \operatorname{SEN}(\Sigma)$, by $\varphi \in C_{\Sigma}^{\mathfrak{\mathfrak { U } ^ { \prime }}}(\Phi)$ if and only if

$$
\alpha_{\Sigma^{\prime}}(\operatorname{SEN}(f)(\Phi)) \subseteq T_{\Sigma^{\prime}}^{\prime} \quad \text { implies } \quad \alpha_{\Sigma^{\prime}}(\operatorname{SEN}(f)(\varphi)) \in T_{\Sigma^{\prime}}^{\prime},
$$

for all $\Sigma^{\prime} \in|\operatorname{Sign}|$ and all $f \in \operatorname{Sign}\left(\Sigma, \Sigma^{\prime}\right)$. It is not difficult, perhaps only a bit tedious, to prove that $C^{\mathfrak{X} \mathfrak{X}^{\prime}}$, thus defined, is a closure system on SEN and, therefore, $d^{\mathfrak{A O}^{\prime}}:=\left\langle\right.$ Sign, SEN, $\left.C^{\mathfrak{A}{ }^{\prime}}\right\rangle$ is a $\pi$-institution.

Proposition $1 \quad$ Let $d=\langle$ Sign, SEN, $C\rangle$ be a $\pi$-institution, with $N$ a category of natural transformations on $\mathrm{SEN}$, let $\mathcal{A}^{\prime}=\left\langle\mathrm{SEN}^{\prime},\langle F, \alpha\rangle\right\rangle$ be an $N$-algebraic system, and let $T^{\prime}=\left\{T_{\Sigma}^{\prime}\right\}_{\Sigma \in\left|\mathbf{S i g n}^{\prime}\right|}$ be an axiom family of $\mathrm{SEN}^{\prime}$ :

$$
T^{\prime} \in \operatorname{ThFam}_{d}^{\langle F, \alpha\rangle}\left(\mathrm{SEN}^{\prime}\right) \quad \text { iff } \quad C \leq C^{\left\langle\mathcal{A}^{\prime}, T^{\prime}\right\rangle} .
$$

Proof Assume, first, that $T^{\prime} \in \operatorname{ThFam}_{\ell}^{\langle F, \alpha\rangle}\left(\mathrm{SEN}^{\prime}\right)$. Let $\Sigma \in \mid$ Sign $\mid$ and $\Phi \cup\{\varphi\} \subseteq \operatorname{SEN}(\Sigma)$, such that $\varphi \in C_{\Sigma}(\Phi)$. Thus, since $d^{\mathcal{A}^{\prime}}$ is an $\left(N, N^{\prime}\right)$-model of d, we get that $\alpha_{\Sigma}(\varphi) \in C_{F(\Sigma)}^{\mathcal{A}^{\prime}}\left(\alpha_{\Sigma}(\Phi)\right)$. Hence, by structurality and the fact that $\alpha$ is a natural transformation, for all $\Sigma^{\prime} \in|\operatorname{Sign}|$ and all $f \in \operatorname{Sign}\left(\Sigma, \Sigma^{\prime}\right)$,

$$
\alpha_{\Sigma^{\prime}}(\operatorname{SEN}(f)(\varphi)) \in C_{F\left(\Sigma^{\prime}\right)}^{\mathcal{A}^{\prime}}\left(\alpha_{\Sigma^{\prime}}(\operatorname{SEN}(f)(\Phi))\right) .
$$


Therefore, since $T^{\prime} \in \operatorname{ThFam}_{\ell}^{\langle F, \alpha\rangle}\left(\operatorname{SEN}^{\prime}\right)$, for all $\Sigma \in|\operatorname{Sign}|$ and all $f \in \operatorname{Sign}\left(\Sigma, \Sigma^{\prime}\right)$,

$$
\alpha_{\Sigma^{\prime}}(\operatorname{SEN}(f)(\Phi)) \subseteq T_{F\left(\Sigma^{\prime}\right)}^{\prime} \quad \text { implies } \quad \alpha_{\Sigma^{\prime}}(\operatorname{SEN}(f)(\varphi)) \in T_{F\left(\Sigma^{\prime}\right)}^{\prime}
$$

This proves that $\varphi \in C_{\Sigma}^{\left\langle\mathcal{A}^{\prime}, T^{\prime}\right\rangle}(\Phi)$, that is, that $C \leq C^{\left\langle\mathcal{A}^{\prime}, T^{\prime}\right\rangle}$.

Conversely, define the collection $C^{\prime}=\left\{C_{\Sigma}^{\prime}\right\}_{\Sigma \in\left|\mathbf{S i g n}^{\prime}\right|}$ by letting, for all $\Sigma \in\left|\mathbf{S i g n}^{\prime}\right|$, $C_{\Sigma}^{\prime}: \mathcal{P}\left(\operatorname{SEN}^{\prime}(\Sigma)\right) \rightarrow \mathcal{P}\left(\operatorname{SEN}^{\prime}(\Sigma)\right)$ given, for all $\Phi \cup\{\varphi\} \subseteq \operatorname{SEN}^{\prime}(\Sigma)$, by $\varphi \in C_{\Sigma}^{\prime}(\Phi)$ iff, for all $\Sigma^{\prime} \in\left|\mathbf{S i g n}^{\prime}\right|$ and all $f \in \operatorname{Sign}^{\prime}\left(\Sigma, \Sigma^{\prime}\right)$,

$$
\operatorname{SEN}^{\prime}(f)(\Phi) \subseteq T_{\Sigma^{\prime}}^{\prime} \quad \text { implies } \operatorname{SEN}^{\prime}(f)(\varphi) \in T_{\Sigma^{\prime}}^{\prime}
$$

for all axiom families $T^{\prime}=\left\{T_{\Sigma}^{\prime}\right\}_{\Sigma \in\left|\mathbf{S i g n}^{\prime}\right|}$ on $\mathrm{SEN}^{\prime}$, such that $C \leq C^{\left\langle\mathcal{A}^{\prime}, T^{\prime}\right\rangle}$. It is not difficult to show that $C^{\prime}$ is a closure system on $\mathrm{SEN}^{\prime}$ and that $d^{\prime}=\left\langle\mathbf{S i g n}^{\prime}, \mathrm{SEN}^{\prime}, C^{\prime}\right\rangle$ is an $\left(N, N^{\prime}\right)$-model of $\ell$. Thus, by the minimality of $C^{\mathcal{A}^{\prime}}$, we get that $C^{\mathcal{A}^{\prime}} \leq C^{\prime}$. This shows that every theory family $T^{\prime}$ of $C^{\prime}$ must also be a theory family of $C^{\mathcal{A}^{\prime}}$. Since, by construction, all axiom families $T^{\prime}$ on $\mathrm{SEN}^{\prime}$, such that $C \leq C^{\left\langle\mathcal{A}^{\prime}, T^{\prime}\right\rangle}$, are theory families of $C^{\prime}$, this establishes the right-to-left implication in the conclusion.

The $N^{\prime}$-Leibniz operator $\Omega^{N^{\prime}}$ of an $N$-algebraic system $\mathcal{A}^{\prime}=\left\langle\mathrm{SEN}^{\prime},\langle F, \alpha\rangle\right\rangle$ associates with every theory family $T^{\prime} \in \mathrm{ThFam}_{\ell}^{\langle F, \alpha\rangle}\left(\mathrm{SEN}^{\prime}\right)$ the largest $N^{\prime}$-congruence system $\Omega^{N^{\prime}}\left(T^{\prime}\right)$ that is compatible with $T^{\prime}$. Similarly, the $N^{\prime}$-Suszko operator $\tilde{\Omega}^{N^{\prime}}$ associates to every theory family $T^{\prime}$ of the closure system $C^{\mathcal{A}^{\prime}}$ on $\mathrm{SEN}^{\prime}$ the largest $N^{\prime}$-congruence system $\tilde{\Omega}^{N^{\prime}}\left(T^{\prime}\right)$ on $\mathrm{SEN}^{\prime}$ that is compatible with every theory family $T^{\prime \prime} \in \operatorname{ThFam}_{\downarrow}^{\langle F, \alpha\rangle}\left(\mathrm{SEN}^{\prime}\right)$, such that $T^{\prime} \leq T^{\prime \prime}$. The $N$-matrix system $\mathfrak{U}=\left\langle\left\langle\mathrm{SEN}^{\prime},\langle F, \alpha\rangle\right\rangle, T^{\prime}\right\rangle$ is $N^{\prime}$-Leibniz reduced if $\Omega^{N^{\prime}}\left(T^{\prime}\right)=\Delta^{\mathrm{SEN}^{\prime}}$, and it is $N^{\prime}$-Suszko reduced if $\tilde{\Omega}^{N^{\prime}}\left(T^{\prime}\right)=\Delta^{\mathrm{SEN}^{\prime}}$.

A $\pi$-institution $d=\langle$ Sign, SEN, $C\rangle$, with $N$ a category of natural transformations on SEN, is said to be syntactically $N$-truth-equational if there exists a collection $\tau$ of $N$-equations, that is, pairs of unary natural transformations $\delta, \epsilon: \mathrm{SEN} \rightarrow \mathrm{SEN}$ in $N$, such that, for every reduced $N$-matrix system model $\mathfrak{U}^{\prime}=\left\langle\mathcal{A}^{\prime}, T^{\prime}\right\rangle$ of $\mathcal{\ell}$, with $\mathcal{A}^{\prime}=\left\langle\mathrm{SEN}^{\prime},\langle F, \alpha\rangle\right\rangle$ an $N$-algebraic system, with $\langle F, \alpha\rangle: \operatorname{SEN} \rightarrow \mathrm{SEN}^{\prime}$ surjective, all $\Sigma \in|\operatorname{Sign}|$, and all $\varphi \in \operatorname{SEN}(\Sigma)$,

$$
\alpha_{\Sigma}(\varphi) \in T_{F(\Sigma)}^{\prime} \quad \text { iff } \quad \delta_{F(\Sigma)}^{\prime}\left(\alpha_{\Sigma}(\varphi)\right)=\epsilon_{F(\Sigma)}^{\prime}\left(\alpha_{\Sigma}(\varphi)\right), \text { for all } \delta \approx \epsilon \in \boldsymbol{\tau} .
$$

On the other hand, $\mathcal{d}$ is said to be (semantically) $N$-truth-equational if the $N$-Leibniz operator $\Omega^{N}$ is completely order reflecting, that is, for all collections of theory families $T^{i} \in \operatorname{ThFam}(\mathcal{l}), i \in I$, and all theory families $T \in \operatorname{ThFam}(\mathcal{l})$,

$$
\bigcap_{i \in I} \Omega^{N}\left(T^{i}\right) \leq \Omega^{N}(T) \text { implies } \bigcap_{i \in I} T^{i} \leq T .
$$

A stronger condition than semantic truth-equationality requires that the Leibniz operator be completely order reflecting on $\operatorname{ThFam}_{\downarrow}^{\langle F, \alpha\rangle}\left(\mathrm{SEN}^{\prime}\right)$, for every $N$-algebraic system $\mathcal{A}^{\prime}=\left\langle\mathrm{SEN}^{\prime},\langle F, \alpha\rangle\right\rangle$, with $\langle F, \alpha\rangle: \mathrm{SEN} \rightarrow \mathrm{SEN}^{\prime}$ a surjective $\left(N, N^{\prime}\right)$-epimorphic translation. This condition turns out to be equivalent to the requirement that the Suszko operator be injective on the lattice of all filters on every $N$-algebraic system $\mathcal{A}^{\prime}=\left\langle\mathrm{SEN}^{\prime},\langle F, \alpha\rangle\right.$, with $\langle F, \alpha\rangle: \mathrm{SEN} \rightarrow \mathrm{SEN}^{\prime}$ a surjective $\left(N, N^{\prime}\right)$-epimorphic translation. It will be shown that syntactic $N$-truthequationality implies this latter condition which, in turn, implies semantic $N$-truth- 
equationality. Moreover, a sufficient condition will be established under which all three conditions turn out to be equivalent. When this condition is applied to the setting of sentential logics, it yields as a corollary one of the main theorems obtained by Raftery in [23].

\section{Leibniz and Suszko Operators}

In this section we recall the definitions and several facts concerning the categorical Leibniz and Suszko operators. The categorical Leibniz operator was defined first in Voutsadakis [28], [29] with the goal of introducing the classes of prealgebraic and protoalgebraic $\pi$-institutions. Its introduction followed the work of Blok and Pigozzi [3] that introduced the Leibniz operator for the first time to characterize algebraizable logics. The categorical Suszko operator was introduced in Voutsadakis [31], taking after the work of Czelakowski [9], who introduced the Suszko operator with the goal of lifting some of the methods of abstract algebraic logic that are applicable to the class of protoalgebraic deductive systems to arbitrary logics.

Let $d=\langle$ Sign, SEN, $C\rangle$ be a $\pi$-institution, with $N$ a category of natural transformations on SEN. Let also $T=\left\{T_{\Sigma}\right\}_{\Sigma \in \mid \text { Sign } \mid}$ be a theory family of SEN. The Leibniz $N$-congruence system $\Omega^{N}(T)$ of $T$ is the largest $N$-congruence system on SEN that is compatible with the theory family $T$. Proposition 2.3 of [29] characterizes Leibniz congruence systems as follows.

Proposition 2 Suppose that $d=\langle\mathbf{S i g n}, \mathrm{SEN}, C\rangle$ is a $\pi$-institution, $N$ is a category of natural transformations on SEN, and $T=\left\{T_{\Sigma}\right\}_{\Sigma \in|\operatorname{Sign}|}$ is a theory family of $d$. Then, for all $\Sigma \in|\operatorname{Sign}|, \varphi, \psi \in \operatorname{SEN}(\Sigma),\langle\varphi, \psi\rangle \in \Omega_{\Sigma}^{N}(T)$ iff, for all $\Sigma^{\prime} \in|\operatorname{Sign}|, f \in \operatorname{Sign}\left(\Sigma, \Sigma^{\prime}\right), \sigma: \operatorname{SEN}^{k} \rightarrow \operatorname{SEN}$ in $N$ and $\vec{\chi} \in \operatorname{SEN}\left(\Sigma^{\prime}\right)^{k}$,

$$
\sigma_{\Sigma^{\prime}}(\operatorname{SEN}(f)(\varphi), \vec{\chi}) \in T_{\Sigma^{\prime}} \quad \text { iff } \quad \sigma_{\Sigma^{\prime}}(\operatorname{SEN}(f)(\psi), \vec{\chi}) \in T_{\Sigma^{\prime}}
$$

Note that in equivalence (3), we have followed a common convention in categorical abstract algebraic logic by which the condition in (3) is a shorthand for the more cumbersome condition: for all $\Sigma^{\prime} \in|\operatorname{Sign}|, f \in \operatorname{Sign}\left(\Sigma, \Sigma^{\prime}\right), \sigma: \operatorname{SEN}^{k} \rightarrow \operatorname{SEN}$ in $N, \vec{\chi} \in \operatorname{SEN}\left(\Sigma^{\prime}\right)^{k}$, and all $i<k$,

$$
\begin{aligned}
& \sigma_{\Sigma^{\prime}}\left(\chi_{0}, \ldots, \chi_{i-1}, \operatorname{SEN}(f)(\varphi), \chi_{i+1}, \ldots, \chi_{k-1}\right) \in T_{\Sigma^{\prime}} \\
& \quad \text { iff } \sigma_{\Sigma^{\prime}}\left(\chi_{0}, \ldots, \chi_{i-1}, \operatorname{SEN}(f)(\psi), \chi_{i+1}, \ldots, \chi_{k-1}\right) \in T_{\Sigma^{\prime}} .
\end{aligned}
$$

Thus, even though there appears to be a mismatch in the declared arity of the natural transformation $\sigma$ and the number of arguments used, this is only apparent, since one of the components of the vector $\vec{\chi}$ employed, when this notation is used, is omitted. This notational convention will be followed throughout the paper, possibly without being mentioned explicitly.

We say that a $\pi$-institution $d=\langle$ Sign, SEN, $C\rangle$ has no theorems if, for all $\Sigma \in|\mathbf{S i g n}|, \operatorname{Thm}_{\Sigma}(\mathcal{l}):=C_{\Sigma}(\emptyset)=\emptyset$.

Lemma $3 \quad$ Let $d=\langle$ Sign, SEN, $C\rangle$ be a $\pi$-institution, with $N$ a category of natural transformations on $\mathrm{SEN}$, such that $\operatorname{SEN}(\Sigma) \neq \emptyset$, for some $\Sigma \in|\operatorname{Sign}|$. If d has no theorems, then the $N$-Leibniz operator is not injective on $\operatorname{ThFam}(\mathcal{d})$. 
Proof Under the hypotheses of the lemma, the collections Thm $=\{\emptyset\}_{\Sigma \in \mid \text { Sign } \mid}$ and $\operatorname{SEN}=\{\operatorname{SEN}(\Sigma)\}_{\Sigma \in|\operatorname{Sign}|}$ are two different theory families of $d$ and, by Proposition 2, we have

$$
\Omega_{\Sigma}^{N}(\mathrm{Thm})=\operatorname{SEN}(\Sigma)^{2}=\Omega_{\Sigma}^{N}(\mathrm{SEN}),
$$

whence $\Omega^{N}(\mathrm{Thm})=\Omega^{N}(\mathrm{SEN})$ and $\Omega^{N}$ is not injective.

Recall from [29] (see also Voutsadakis [33]) that, given two $\pi$-institutions $d=$ $\langle$ Sign, SEN,$C\rangle$ and $l^{\prime}=\left\langle\right.$ Sign $\left.^{\prime}, \mathrm{SEN}^{\prime}, C^{\prime}\right\rangle$, an $\left(N, N^{\prime}\right)$-logical morphism (also known as an $\left(N, N^{\prime}\right)$-epimorphic semiinterpretation) $\langle F, \alpha\rangle: \ell \succ \ell^{\prime}$, and a theory family $T^{\prime} \in \operatorname{ThFam}\left(d^{\prime}\right)$, the theory family $\alpha^{-1}\left(T^{\prime}\right)$ of $d$ is defined by setting

$$
\alpha^{-1}\left(T^{\prime}\right)=\left\{\alpha_{\Sigma}^{-1}\left(T_{F(\Sigma)}^{\prime}\right)\right\}_{\Sigma \in \mid \text { Sign| }} .
$$

Lemma 5.4 of [29] shows that, roughly speaking, the Leibniz operator commutes with inverse surjective logical morphisms.

Lemma $4 \quad$ Let $d=\langle$ Sign, SEN, $C\rangle$ be a $\pi$-institution, let $N$ be a category of natural transformations on $\mathrm{SEN}$, and let $d^{\prime}=\left\langle\mathbf{S i g n}^{\prime}, \mathrm{SEN}^{\prime}, C^{\prime}\right\rangle$ be an $\left(N, N^{\prime}\right)$-model of $d$ via a surjective $\left(N, N^{\prime}\right)$-logical morphism $\left.\langle F, \alpha\rangle: d\right\rangle-d^{\prime}$. Then, for every theory family $T^{\prime}$ of $d^{\prime}$ and every $\Sigma \in|\mathbf{S i g n}|, \Omega_{\Sigma}^{N}\left(\alpha^{-1}\left(T^{\prime}\right)\right)=\alpha_{\Sigma}^{-1}\left(\Omega_{F(\Sigma)}^{N^{\prime}}\left(T^{\prime}\right)\right)$.

Sometimes, the relation in the conclusion of Lemma 4 is denoted by

$$
\Omega^{N}\left(\alpha^{-1}\left(T^{\prime}\right)\right)=\alpha^{-1}\left(\Omega^{N^{\prime}}\left(T^{\prime}\right)\right) .
$$

One important class of $\pi$-institutions from the point of view of categorical abstract algebraic logic is the class of (semantically) $N$-protoalgebraic $\pi$-institutions (see [29]). These are the $\pi$-institutions that have Leibniz operators that are monotonic on the lattice of all their theory families. For a long time it had been suggested that the class of protoalgebraic deductive systems is the widest class reasonably amenable to treatment by universal algebraic methods and techniques (see [3], [12]). More recently, however, as more natural examples of nonprotoalgebraic logics have been discovered, there has been some effort to expand the methods of abstract algebraic logic to be able to handle nonprotoalgebraic deductive systems. Czelakowski [9] (see also [8] and [11]) has suggested that the viable alternative to the Leibniz operator that may need to be considered in the study of nonprotoalgebraic logics is the Suszko operator. Given a $\pi$-institution $d=\langle\operatorname{Sign}, \operatorname{SEN}, C\rangle$, with a category $N$ of natural transformations on SEN, the $N$-Suszko operator $\tilde{\Omega}^{N}$ associates with every theory family $T$ of $d$ the signature wise intersection of all Leibniz congruence systems of all theory families that contain $T$. It is easy to see that for $N$-protoalgebraic $\pi$-institutions the $N$-Leibniz operator and the $N$-Suszko operator coincide. The $N$-Suszko operator, however, is monotone on the theory families of a $\pi$-institution even when the $\pi$-institution is not $N$-protoalgebraic. Along the lines of switching from the Leibniz to the Suszko operator for the study of arbitrary deductive systems, one may consider the class of Suszko-reduced $\mathcal{\ell}$-matrix systems $\mathrm{M}^{\mathrm{Su}}(\mathcal{\ell})$ (corresponding to the class $\operatorname{Mod}^{\mathrm{Su}} \delta$ of all Suszko-reduced matrices of a deductive system 8 ) instead of the class $\mathrm{M}^{*}(\mathcal{l})$ (corresponding to $\mathrm{Mod}^{*} \&$ ) of Leibniz-reduced ones.

To provide more details, recall from [29, Section 6] (see also [31]) that given a $\pi$-institution $d=\langle\operatorname{Sign}, \operatorname{SEN}, C\rangle$, with $N$ a category of natural transformations on SEN, and a theory family $T=\left\{T_{\Sigma}\right\}_{\Sigma \in \mid \text { Sign } \mid}$ of $d$, the family of binary relations 
$\tilde{\Omega}^{N}(T)=\left\{\tilde{\Omega}_{\Sigma}^{N}(T)\right\}_{\Sigma \in|\operatorname{Sign}|}$ defined by letting, for all $\Sigma \in|\operatorname{Sign}|, \varphi, \psi \in \operatorname{SEN}(\Sigma)$, $\langle\varphi, \psi\rangle \in \tilde{\Omega}_{\Sigma}^{N}(T)$ iff

$$
C_{\Sigma^{\prime}}\left(T_{\Sigma^{\prime}} \cup\left\{\sigma_{\Sigma^{\prime}}(\operatorname{SEN}(f)(\varphi), \vec{\chi})\right\}\right)=C_{\Sigma^{\prime}}\left(T_{\Sigma^{\prime}} \cup\left\{\sigma_{\Sigma^{\prime}}(\operatorname{SEN}(f)(\psi), \vec{\chi})\right\}\right),
$$

for all $\Sigma^{\prime} \in|\operatorname{Sign}|, f \in \operatorname{Sign}\left(\Sigma, \Sigma^{\prime}\right), \sigma: \operatorname{SEN}^{k} \rightarrow \operatorname{SEN}$ in $N$ and $\vec{\chi} \in \operatorname{SEN}\left(\Sigma^{\prime}\right)^{k}$, defines an $N$-congruence system on SEN that is compatible with the theory family $T$, called the Suszko $N$-congruence system of $T$.

Proposition 6.3 of [29] asserts that the $N$-Suszko operator, unlike the $N$-Leibniz operator, and similarly with the deductive system framework, is always monotone on theory families of a $\pi$-institution.

Proposition $5 \quad$ Let $d=\langle$ Sign, SEN, $C\rangle$ be a $\pi$-institution, with $N$ a category of natural transformations on SEN. Then $\tilde{\Omega}^{N}\left(T^{1}\right) \leq \tilde{\Omega}^{N}\left(T^{2}\right)$, for all theory families $T^{1}, T^{2}$ of $\mathcal{d}$, such that $T^{1} \leq T^{2}$.

Finally, [29, Theorem 6.4] characterizes the Suszko operator as follows.

Theorem $6 \quad$ Let $d=\langle$ Sign, SEN, $C\rangle$ be a $\pi$-institution, with $N$ a category of natural transformations on SEN. Suppose that, for every theory family $T$ of $\mathcal{l}, \mathcal{O}^{N}(T)$ is an $N$-congruence system on $\operatorname{SEN}$, such that, for all $\Sigma \in|\operatorname{Sign}|, \varphi, \psi \in \operatorname{SEN}(\Sigma)$,

$$
\langle\varphi, \psi\rangle \in \mathcal{O}_{\Sigma}^{N}(T) \quad \text { implies } \quad C_{\Sigma}\left(T_{\Sigma} \cup\{\varphi\}\right)=C_{\Sigma}\left(T_{\Sigma} \cup\{\psi\}\right) .
$$

Then $\mathcal{O}^{N}(T) \leq \tilde{\Omega}^{N}(T)$, for all theory families $T$ of $\mathcal{l}$.

Recall from [29, Section 3] the definition of the least theory family of a $\pi$-institution $\mathcal{d}=\langle$ Sign, SEN, $C\rangle$ containing a given theory family $T \in \operatorname{ThFam}(\mathcal{L})$ and a given $\varphi \in \operatorname{SEN}(\Sigma)$. It is denoted by $T^{[\langle\Sigma, \varphi\rangle]}=\left\{T_{\Sigma^{\prime}}^{[\langle\Sigma, \varphi\rangle]}\right\}_{\Sigma^{\prime} \in|\operatorname{Sign}|}$ and defined, for all $\Sigma^{\prime} \in|\mathbf{S i g n}|$, by

$$
T_{\Sigma^{\prime}}^{[\langle\Sigma, \varphi\rangle]}= \begin{cases}T_{\Sigma^{\prime}} & \text { if } \Sigma^{\prime} \neq \Sigma, \\ C_{\Sigma}\left(T_{\Sigma} \cup\{\varphi\}\right) & \text { if } \Sigma^{\prime}=\Sigma .\end{cases}
$$

Recall, also, the notation Thm $=\left\{\operatorname{Thm}_{\Sigma}\right\}_{\Sigma \in \mid \text { Sign } \mid}$ for the theorem system of $d$, that is, $\operatorname{Thm}_{\Sigma}=C_{\Sigma}(\emptyset)$, for all $\Sigma \in|\mathbf{S i g n}|$. Finally, recall the related notion of the least theory system $T^{\langle\Sigma, \varphi\rangle}$ of $d$ generated by a given theory system $T$ of $d$ and a given $\varphi \in \operatorname{SEN}(\Sigma)$ (see [29, Lemma 3.1]).

The following proposition, which we perceive as an analogue of [23, Proposition 8] in the present context, establishes conditions under which, roughly speaking, the substitution instances $\tau_{\Sigma}(\varphi)$ of a set of $N$-equations $\tau$ by a given $\Sigma$-sentence $\varphi$ belong to the $\Sigma$-component $\tilde{\Omega}_{\Sigma}^{N}\left(\mathrm{Thm}^{[\langle\Sigma, \varphi\rangle]}\right)$ of the Suszko congruence system $\tilde{\Omega}^{N}\left(\mathrm{Thm}^{[\langle\Sigma, \varphi\rangle]}\right)$ of the theory family $\mathrm{Thm}^{[\langle\Sigma, \varphi\rangle]}$ generated by the given $\Sigma$-sentence $\varphi$.

Proposition $7 \quad$ Let $d=\langle$ Sign, SEN, $C\rangle$ be a $\pi$-institution, with $N$ a category of natural transformations on SEN. Also, let $\boldsymbol{\tau}$ be a collection of pairs $\langle\delta, \epsilon\rangle$ of natural transformations $\delta, \epsilon: \mathrm{SEN} \rightarrow \mathrm{SEN}$ in $N$. Then, for all $\Sigma \in|\mathbf{S i g n}|$ and all $\varphi \in \operatorname{SEN}(\Sigma)$, the following statements are equivalent:

1. for every $\sigma: \operatorname{SEN}^{k} \rightarrow \operatorname{SEN}$ in $N$, all $\Sigma^{\prime} \in|\operatorname{Sign}|$, all $f \in \operatorname{Sign}\left(\Sigma, \Sigma^{\prime}\right)$, and all $\vec{\chi} \in \operatorname{SEN}\left(\Sigma^{\prime}\right)^{k}$,

$$
\begin{aligned}
& C_{\Sigma^{\prime}}\left(\operatorname{Thm}_{\Sigma^{\prime}}^{[\langle\Sigma, \varphi\rangle]}, \sigma_{\Sigma^{\prime}}\left(\operatorname{SEN}(f)\left(\delta_{\Sigma}(\varphi)\right), \vec{\chi}\right)\right) \\
& \quad=C_{\Sigma^{\prime}}\left(\operatorname{Thm}_{\Sigma^{\prime}}^{[\langle\Sigma, \varphi\rangle]}, \sigma_{\Sigma^{\prime}}\left(\operatorname{SEN}(f)\left(\epsilon_{\Sigma}(\varphi)\right), \vec{\chi}\right)\right),
\end{aligned}
$$


for all $\langle\delta, \epsilon\rangle \in \boldsymbol{\tau}$;

2. $\tau_{\Sigma}(\varphi) \subseteq \tilde{\Omega}_{\Sigma}^{N}(T)$, for all $T \in \operatorname{ThFam}(\mathcal{Q})$, such that $\varphi \in T_{\Sigma}$;

3. $\tau_{\Sigma}(\varphi) \subseteq \tilde{\Omega}_{\Sigma}^{N}\left(\operatorname{Thm}^{[\langle\Sigma, \varphi\rangle]}\right)$.

\section{Proof}

(1) $\leftrightarrow$ (3) This equivalence follows directly from the definition of the Suszko $N$-congruence system corresponding to $\mathrm{Thm}^{[\langle\Sigma, \varphi\rangle]}$ (see equation (4)).

(2) $\leftrightarrow$ (3) The left-to-right implication follows easily by replacing $T$ by $\operatorname{Thm}^{[\langle\Sigma, \varphi\rangle]}$ and observing that $\varphi \in \operatorname{Thm}_{\Sigma}^{[\langle\Sigma, \varphi\rangle]}$. The converse follows by noting that, for every $T \in \operatorname{ThFam}(\mathcal{Q})$, if $\varphi \in T_{\Sigma}$, then $\operatorname{Thm}^{[\langle\Sigma, \varphi\rangle]} \leq T$ and taking into account the monotonicity of $\tilde{\Omega}^{N}$ on $\operatorname{ThFam}(\mathcal{l})$.

Let $d=\langle$ Sign, SEN, $C\rangle$ be a $\pi$-institution, with $N$ a category of natural transformations on SEN; $\boldsymbol{\tau}$ an $N$-translation, that is, a set of pairs $\delta \approx \epsilon$ of unary natural transformations $\delta, \epsilon: \mathrm{SEN} \rightarrow \mathrm{SEN}$ in $N$; and $\mathfrak{F}=\left\{\left\langle\operatorname{SEN}^{i},\left\langle F^{i}, \alpha^{i}\right\rangle\right\rangle: i \in I\right\}$ a collection of $N$-algebraic systems. Recall from Voutsadakis [32] the definition of the closure system $C \widetilde{\mho}$ on $\mathrm{SEN}^{2}$ generated by the class $\mathfrak{F}$. Moreover, recall that if $\mathfrak{F}$ is a class of $\boldsymbol{\tau}$-algebraic models of $\mathcal{d}$, it is called a $\tau$-algebraic semantics of $\mathcal{d}$ if, for all $\Sigma \in|\operatorname{Sign}|, \Phi \cup\{\varphi\} \subseteq \operatorname{SEN}(\Sigma)$,

$$
\varphi \in C_{\Sigma}(\Phi) \quad \text { iff } \quad \tau_{\Sigma}(\varphi) \subseteq C_{\Sigma}^{\mathfrak{F}}\left(\tau_{\Sigma}(\Phi)\right)
$$

The following necessary condition for the existence of an algebraic semantics for a $\pi$-institution is proven in [32, Corollary 8.3].

Corollary $8 \quad$ Let $d=\langle$ Sign, SEN, $C\rangle$ be a $\pi$-institution, with $N$ a category of natural transformations on SEN, having a $\boldsymbol{\tau}$-algebraic semantics $\mathfrak{F}$, where $\tau$ is an $N$-translation. Then, for all $T \in \operatorname{ThFam}(\mathcal{\ell})$, all $\Sigma \in|\operatorname{Sign}|$, and all $\varphi \in \operatorname{SEN}(\Sigma)$, such that $\operatorname{SEN}(f)(\varphi) \in T_{\Sigma^{\prime}}$, for all $\Sigma^{\prime} \in|\operatorname{Sign}|$ and all $f \in \operatorname{Sign}\left(\Sigma, \Sigma^{\prime}\right)$, we have $\tau_{\Sigma}(\varphi) \subseteq \Omega_{\Sigma}^{N}(T)$.

Let $d=\langle$ Sign, SEN, $C\rangle$ be a $\pi$-institution, with $N$ a category of natural transformations on SEN. Let $\mathrm{SEN}^{\prime}:$ Sign' $^{\prime} \rightarrow$ Set be a functor, with $N^{\prime}$ a category of natural transformations on $\mathrm{SEN}^{\prime}$, and let $\langle F, \alpha\rangle: \mathrm{SEN} \rightarrow \mathrm{SEN}^{\prime}$ be a surjective $\left(N, N^{\prime}\right)$-epimorphic translation. Let us denote by $d^{\langle F, \alpha\rangle}=\left\langle\mathbf{S i g n}^{\prime}, \mathrm{SEN}^{\prime}, C^{\langle F, \alpha\rangle}\right\rangle$ the $\langle F, \alpha\rangle$-min $\left(N, N^{\prime}\right)$-model of $d$ on $\operatorname{SEN}^{\prime}$, and set $\operatorname{ThFam}\left(\mathcal{d}^{\langle F, \alpha\rangle}\right)=$ $\mathrm{ThFam}_{d}^{\langle F, \alpha\rangle}\left(\mathrm{SEN}^{\prime}\right)$, the collection of all theory families of $d^{\langle F, \alpha\rangle}$. Proposition 1 of Section 2 dealt with this $\pi$-institution and its theory families. We say that the Leibniz or the Suszko operator of $d$ is globally injective if the Leibniz or the Suszko operator, respectively, is injective on $\operatorname{ThFam}\left(\mathcal{d}^{\langle F, \alpha\rangle}\right)$, for every surjective $\left(N, N^{\prime}\right)$-epimorphic translation $\langle F, \alpha\rangle: \mathrm{SEN} \rightarrow \mathrm{SEN}^{\prime}$.

The following theorem forms an analogue of Czelakowski's [9, Theorem 7.8] (see also [23, Theorem 10]) that provides a characterization of the global injectivity of the Suszko operator of a deductive system.

Theorem 9 (Czelakowski) Let $d=\langle$ Sign, SEN, $C\rangle$ be a $\pi$-institution, with $N$ a category of natural transformations on SEN. The following conditions are equivalent.

(i) The N-Suszko operator of d is globally injective. 
(ii) For every surjective $\left(N, N^{\prime}\right)$-epimorphic translation $\langle F, \alpha\rangle: \mathrm{SEN} \rightarrow \mathrm{SEN}^{\prime}$ and every theory family $T \in \operatorname{ThFam}\left(\mathcal{Q}^{\langle F, \alpha\rangle}\right)$,

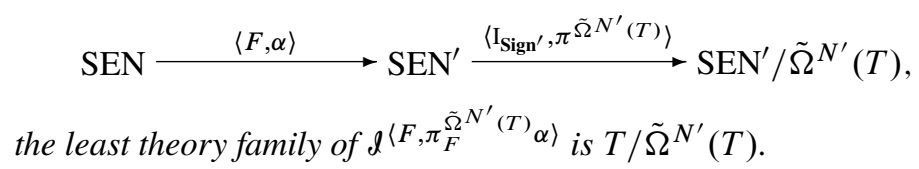

Proof Assume, first, that the $N$-Suszko operator of $\mathcal{l}$ is globally injective, and let $T^{\prime}$ be the least theory family in $\operatorname{ThFam}\left(\mathcal{Q}^{\left\langle F, \pi_{F}^{\tilde{\Omega}^{N^{\prime}}(T)} \alpha\right\rangle}\right)$. We will show that $T^{\prime}=T / \tilde{\Omega}^{N^{\prime}}(T)$. Since $\tilde{\Omega}^{N^{\prime}}(T)$ is compatible with $T$ and $T \in \operatorname{ThFam}\left(\mathcal{d}^{\langle F, \alpha\rangle}\right)$, $T / \tilde{\Omega}^{N^{\prime}}(T)$ is a theory family of $d^{\left\langle F, \pi_{F}^{N^{N^{\prime}}(T)} \alpha\right\rangle}$, whence, by the postulated minimality of $T^{\prime}, T^{\prime} \leq T / \tilde{\Omega}^{N^{\prime}}(T)$. Now, taking into account the monotonicity of $\tilde{\Omega}^{N^{\prime \Omega^{N^{\prime}}(T)}}$, we get the following chain of inclusions:

$$
\begin{aligned}
\Delta^{\operatorname{SEN}^{\prime \tilde{\Omega}^{N^{\prime}}(T)}} & \leq \tilde{\Omega}^{N^{\prime \tilde{\Omega}^{N^{\prime}}(T)}}\left(T^{\prime}\right) \\
& \leq \tilde{\Omega}^{N^{\prime \tilde{\Omega}^{N^{\prime}}(T)}}\left(T / \tilde{\Omega}^{N^{\prime}}(T)\right) \\
& =\Delta^{\operatorname{SEN}^{\prime \tilde{\Omega}^{N^{\prime}}(T)}} .
\end{aligned}
$$

Therefore, $\tilde{\Omega}^{N^{\prime \Omega^{N^{\prime}}(T)}}\left(T^{\prime}\right)=\tilde{\Omega}^{N^{\prime \tilde{\Omega}^{N^{\prime}}(T)}}\left(T / \tilde{\Omega}^{N^{\prime}}(T)\right)$, and hence, by injectivity, we obtain $T^{\prime}=T / \tilde{\Omega}^{N^{\prime}}(T)$.

Assume, conversely, that $\langle F, \alpha\rangle: \operatorname{SEN} \rightarrow \operatorname{SEN}^{\prime}$ is a surjective $\left(N, N^{\prime}\right)$ epimorphic translation and $T, T^{\prime} \in \operatorname{ThFam}\left(\mathcal{Q}^{\langle F, \alpha\rangle}\right)$, such that $\tilde{\Omega}^{N^{\prime}}(T)=\tilde{\Omega}^{N^{\prime}}\left(T^{\prime}\right)$. Consider the two functors $\operatorname{SEN}^{\prime \tilde{\Omega}^{N^{\prime}}(T)}$ and $\operatorname{SEN}^{\prime \tilde{\Omega}^{N^{\prime}}\left(T^{\prime}\right)}$. By the hypothesis, the two theory families $T / \tilde{\Omega}^{N^{\prime}}(T)$ and $T^{\prime} / \tilde{\Omega}^{N^{\prime}}\left(T^{\prime}\right)$ are the least theory families on $\operatorname{SEN}^{\prime} \tilde{\Omega}^{N^{\prime}}(T)$ and $\operatorname{SEN}^{\prime} \tilde{\Omega}^{N^{\prime}}\left(T^{\prime}\right)$, respectively. But, since $\tilde{\Omega}^{N^{\prime}}(T)=\tilde{\Omega}^{N^{\prime}}\left(T^{\prime}\right)$, these two functors coincide, as do the corresponding canonical projections, implying that $T / \tilde{\Omega}^{N^{\prime}}(T)=T^{\prime} / \tilde{\Omega}^{N^{\prime}}\left(T^{\prime}\right)$. Again, taking into account that $\tilde{\Omega}^{N^{\prime}}(T)=\tilde{\Omega}^{N^{\prime}}\left(T^{\prime}\right)$, we get that $T=T^{\prime}$.

We say that the Leibniz operator of a $\pi$-institution $d=\langle$ Sign, SEN, $C\rangle$, with $N$ a category of natural transformations on SEN, is globally completely order reflecting if, for every surjective $\left(N, N^{\prime}\right)$-epimorphic translation $\langle F, \alpha\rangle: \mathrm{SEN} \rightarrow \mathrm{SEN}^{\prime}$ and all $\mathcal{T} \cup\left\{T^{\prime}\right\} \subseteq \operatorname{ThFam}\left(\mathcal{Q}^{\langle F, \alpha\rangle}\right)$,

$$
\bigcap_{T \in \mathcal{T}} \Omega^{N^{\prime}}(T) \leq \Omega^{N^{\prime}}\left(T^{\prime}\right) \text { implies } \bigcap_{T \in \mathcal{T}} T \leq T^{\prime} .
$$

In the following proposition an alternative characterization of global complete order reflexivity is provided, involving both the Leibniz and the Suszko operators of a $\pi$-institution $d$. This abstracts condition (5), following [23, Theorem 10], which is applicable for sentential logics.

Proposition $10 \quad$ Let $d=\langle$ Sign, SEN, $C\rangle$ be a $\pi$-institution, with $N$ a category of natural transformations on SEN. The Leibniz operator of d is globally completely order reflecting if and only if, for every surjective $\left(N, N^{\prime}\right)$-epimorphic translation and all $T, T^{\prime} \in \operatorname{ThFam}\left(\mathcal{Q}^{\langle F, \alpha\rangle}\right)$,

$$
\tilde{\Omega}^{N^{\prime}}(T) \leq \Omega^{N^{\prime}}\left(T^{\prime}\right) \quad \text { implies } \quad T \leq T^{\prime} .
$$


Proof Assume, first, that the Leibniz operator is globally completely order reflecting and that, for some surjective $\left(N, N^{\prime}\right)$-epimorphic translation $\langle F, \alpha\rangle: \operatorname{SEN} \rightarrow$ $\operatorname{SEN}^{\prime}, T, T^{\prime} \in \operatorname{ThFam}\left(\mathcal{Q}^{\langle F, \alpha\rangle}\right)$ are such that $\tilde{\Omega}^{N^{\prime}}(T) \leq \Omega^{N^{\prime}}\left(T^{\prime}\right)$. Then, we have

$$
\bigcap_{T \leq T^{\prime \prime}} \Omega^{N^{\prime}}\left(T^{\prime \prime}\right)=\tilde{\Omega}^{N^{\prime}}(T) \leq \Omega^{N^{\prime}}\left(T^{\prime}\right) .
$$

So, by the hypothesis, we get that $\bigcap_{T \leq T^{\prime \prime}} T^{\prime \prime} \leq T^{\prime}$, whence $T \leq T^{\prime}$.

Assume, conversely, that, for every surjective $\left(N, N^{\prime}\right)$-epimorphic translation $\langle F, \alpha\rangle: \mathrm{SEN} \rightarrow \mathrm{SEN}^{\prime}$, and all $T, T^{\prime} \in \operatorname{ThFam}\left(\mathcal{d}^{\langle F, \alpha\rangle}\right)$,

$$
\tilde{\Omega}^{N^{\prime}}(T) \leq \Omega^{N^{\prime}}\left(T^{\prime}\right) \text { implies } T \leq T^{\prime} .
$$

Let $\mathcal{T} \cup\left\{T^{\prime}\right\} \subseteq \operatorname{ThFam}\left(\mathcal{Q}^{\langle F, \alpha\rangle}\right)$, such that $\bigcap_{T \in \mathcal{T}} \Omega^{N^{\prime}}(T) \leq \Omega^{N^{\prime}}\left(T^{\prime}\right)$. Then, we have

$$
\bigcap_{\cap \mathcal{T} \leq T^{\prime \prime}} \Omega^{N^{\prime}}\left(T^{\prime \prime}\right) \leq \bigcap_{T \in \mathcal{T}} \Omega^{N^{\prime}}(T) \leq \Omega^{N^{\prime}}\left(T^{\prime}\right),
$$

that is, $\tilde{\Omega}^{N^{\prime}}(\bigcap \mathcal{T}) \leq \Omega^{N^{\prime}}\left(T^{\prime}\right)$. Therefore, by the hypothesis, $\bigcap \mathcal{T} \leq T^{\prime}$.

The following proposition establishes that the complete order reflexivity of the Leibniz operator of a $\pi$-institution $d$ implies the order reflexivity of both the Leibniz and the Suszko operators of $\mathscr{\ell}$. Since every order-reflecting function between order sets is also injective, Proposition 11 implies that when the Leibniz operator of a $\pi$-institution is completely order reflecting, then both the Leibniz and the Suszko operators are injective.

Proposition 11 Let $d=\langle$ Sign, SEN, $C\rangle$ be a $\pi$-institution, with $N$ a category of natural transformations on SEN. If the Leibniz operator of $d$ is globally completely order reflecting and $\langle F, \alpha\rangle: \mathrm{SEN} \rightarrow \mathrm{SEN}^{\prime}$ is a surjective $\left(N, N^{\prime}\right)$-epimorphic translation, then, for all $T, T^{\prime} \in \operatorname{ThFam}\left(\mathcal{Q}^{\langle F, \alpha\rangle}\right)$, if $\Omega^{N^{\prime}}(T) \leq \Omega^{N^{\prime}}\left(T^{\prime}\right)$ or $\tilde{\Omega}^{N^{\prime}}(T) \leq \tilde{\Omega}^{N^{\prime}}\left(T^{\prime}\right)$, then $T \leq T^{\prime}$.

Proof If $\Omega^{N^{\prime}}(T) \leq \Omega^{N^{\prime}}\left(T^{\prime}\right)$, then, we have $\tilde{\Omega}^{N^{\prime}}(T) \leq \Omega^{N^{\prime}}\left(T^{\prime}\right)$, whence, by the hypothesis and Proposition 10,T $\leq T^{\prime}$. If, on the other hand, $\tilde{\Omega}^{N^{\prime}}(T) \leq \tilde{\Omega}^{N^{\prime}}\left(T^{\prime}\right)$, then $\tilde{\Omega}^{N^{\prime}}(T) \leq \tilde{\Omega}^{N^{\prime}}\left(T^{\prime}\right) \leq \Omega^{N^{\prime}}\left(T^{\prime}\right)$, whence $T \leq T^{\prime}$.

Corollary $12 \quad$ Let $d=\langle$ Sign, SEN, $C\rangle$ be a $\pi$-institution, with $N$ a category of natural transformations on SEN. If the $N$-Leibniz operator of $d$ is globally completely order reflecting, then for every surjective $\left(N, N^{\prime}\right)$-epimorphic translation $\langle F, \alpha\rangle: \mathrm{SEN} \rightarrow \mathrm{SEN}^{\prime}$, both $\Omega^{N^{\prime}}$ and $\tilde{\Omega}^{N^{\prime}}$ are injective on $\operatorname{ThFam}\left(\mathcal{d}^{\langle F, \alpha\rangle}\right)$.

Finally, we close this section by establishing a converse of Corollary 12 to the effect that the global injectivity of the Suszko operator implies the global complete order reflexivity of the Leibniz operator of a given $\pi$-institution. This is an analogue in the categorical framework of [23, Theorem 11].

Theorem 13 Let $d=\langle$ Sign, SEN, $C\rangle$ be a $\pi$-institution, with $N$ a category of natural transformations on SEN. The Suszko operator of $d$ is globally injective if and only if the Leibniz operator is globally completely order reflecting.

Proof If the Leibniz operator of $d$ is globally completely order reflecting, then, by Corollary 12 , the Suszko operator of $d$ is globally injective. 
Suppose, conversely, that the Suszko operator of $d$ is globally injective, that is, that for every surjective $\left(N, N^{\prime}\right)$-epimorphic translation $\langle F, \alpha\rangle: \mathrm{SEN} \rightarrow \mathrm{SEN}^{\prime}$ and all $T, T^{\prime} \in \operatorname{ThFam}\left(\mathcal{Q}^{\langle F, \alpha\rangle}\right), \tilde{\Omega}^{N^{\prime}}(T)=\tilde{\Omega}^{N^{\prime}}\left(T^{\prime}\right)$ implies that $T=T^{\prime}$. To show that the Leibniz operator is globally completely order reflecting, we use Proposition 10. Consider a surjective $\left(N, N^{\prime}\right)$-epimorphic translation $\langle F, \alpha\rangle: \mathrm{SEN} \rightarrow \mathrm{SEN}^{\prime}$ and $T, T^{\prime} \in \operatorname{ThFam}\left(\mathcal{Q}^{\langle F, \alpha\rangle}\right)$, such that $\tilde{\Omega}^{N^{\prime}}(T) \leq \Omega^{N^{\prime}}\left(T^{\prime}\right)$. Then, for all $\Sigma \in\left|\mathbf{S i g n}^{\prime}\right|$, the map $\varphi / \tilde{\Omega}_{\Sigma}^{N^{\prime}}(T) \stackrel{\pi_{\Sigma}}{\mapsto} \varphi / \Omega_{\Sigma}^{N^{\prime}}\left(T^{\prime}\right)$ defines a surjective translation

$$
\left\langle\mathrm{I}_{\mathrm{Sign}^{\prime}}, \pi\right\rangle: \mathrm{SEN}^{\prime \tilde{\Omega}^{N^{\prime}}(T)} \rightarrow \mathrm{SEN}^{\prime \Omega^{N^{\prime}}\left(T^{\prime}\right)} .
$$

Moreover, by the defining property of the Leibniz $N^{\prime}$-congruence system $\Omega^{N^{\prime}}\left(T^{\prime}\right)$ of $T^{\prime}$ and the hypothesis, we obtain that $\tilde{\Omega}^{N^{\prime}}(T)$ is compatible with $T^{\prime}$, whence the notation $T^{\prime} / \tilde{\Omega}^{N^{\prime}}(T)$ may be unambiguously used for the collection $\left\{\varphi / \tilde{\Omega}_{\Sigma}^{N^{\prime}}(T): \varphi \in\right.$ $\left.T_{\Sigma}^{\prime}\right\}_{\Sigma \in \mid \text { Sign }^{\prime} \mid}$. Since, obviously, $\left.T^{\prime} / \Omega^{N^{\prime}}\left(T^{\prime}\right) \in \operatorname{ThFam}\left(\mathcal{Q}^{\left\langle F, \pi_{F}^{\Omega^{N^{\prime}}}\left(T^{\prime}\right)\right.} \alpha\right\rangle\right)$,

$$
\mathrm{SEN} \stackrel{\langle F, \alpha\rangle}{\longrightarrow} \mathrm{SEN}^{\prime} \stackrel{\left\langle\mathrm{I}_{\mathrm{Sign}^{\prime}}, \pi^{\Omega^{N^{\prime}}\left(T^{\prime}\right)}\right\rangle}{\longrightarrow} \operatorname{SEN}^{\prime} / \Omega^{N^{\prime}}\left(T^{\prime}\right)
$$

we get that $\left.\pi^{-1}\left(T^{\prime} / \Omega^{N^{\prime}}\left(T^{\prime}\right)\right)=T^{\prime} / \tilde{\Omega}^{N^{\prime}}(T) \in \operatorname{ThFam}\left(\mathcal{Q}^{\left\langle F, \pi_{F}^{\tilde{\Omega}^{N^{\prime}}}(T)\right.} \alpha\right\rangle\right)$. But, by Theorem $9, T / \tilde{\Omega}^{N^{\prime}}(T)$ is the least theory family of this collection. Therefore $T / \tilde{\Omega}^{N^{\prime}}(T) \leq T^{\prime} / \tilde{\Omega}^{N^{\prime}}(T)$. Thus, if $\varphi \in T_{\Sigma}$, there exists $\varphi^{\prime} \in T_{\Sigma}^{\prime}$, such that $\left\langle\varphi, \varphi^{\prime}\right\rangle \in \tilde{\Omega}_{\Sigma}^{N^{\prime}}(T)$, whence, by the asserted compatibility of $\tilde{\Omega}^{N^{\prime}}(T)$ with $T^{\prime}$, we get that $\varphi \in T_{\Sigma}^{\prime}$. Thus, $T_{\Sigma} \subseteq T_{\Sigma}^{\prime}$. Since this holds for all $\Sigma \in\left|\mathbf{S i g n}^{\prime}\right|$, we get that $T \leq T^{\prime}$.

\section{Definability of Truth}

Let $d=\langle$ Sign, SEN, $C\rangle$ be a $\pi$-institution, with $N$ a category of natural transformations on SEN. We remind the reader that an $N$-matrix system (model) $\left\langle\left\langle\mathrm{SEN}^{\prime},\langle F, \alpha\rangle\right\rangle, T\right\rangle$ of $d$ consists of a functor $\mathrm{SEN}^{\prime}:$ Sign' $\rightarrow$ Set, with $N^{\prime}$ a category of natural transformations on $\mathrm{SEN}^{\prime}$, a surjective $\left(N, N^{\prime}\right)$-epimorphic translation $\langle F, \alpha\rangle: \mathrm{SEN} \rightarrow \mathrm{SEN}^{\prime}$, and a theory family $T \in \operatorname{ThFam}\left(\mathcal{\alpha}^{\langle F, \alpha\rangle}\right)$. Let $\mathrm{M}$ be a class of $N$-matrix systems of $\ell$. We say that truth is implicitly definable in $\mathrm{M}$ if, whenever $\left\langle\left\langle\mathrm{SEN}^{\prime},\langle F, \alpha\rangle\right\rangle, T\right\rangle,\left\langle\left\langle\mathrm{SEN}^{\prime},\langle F, \alpha\rangle\right\rangle, T^{\prime}\right\rangle \in \mathrm{M}$, then $T=T^{\prime}$. On the other hand, we say that truth is equationally definable in $\mathrm{M}$ if there exists an $N$-translation $\tau$, that is, a collection of pairs $\langle\delta, \epsilon\rangle$ of natural transformations $\delta, \epsilon: \operatorname{SEN} \rightarrow$ SEN in $N$, such that, for all $\left\langle\left\langle\operatorname{SEN}^{\prime},\langle F, \alpha\rangle\right\rangle, T\right\rangle \in \mathrm{M}$, all $\Sigma \in\left|\operatorname{Sign}^{\prime}\right|$, and all $\varphi \in \operatorname{SEN}^{\prime}(\Sigma)$,

$$
\begin{gathered}
\left(\forall f \in \operatorname{Sign}^{\prime}\left(\Sigma, \Sigma^{\prime}\right)\right)\left(\operatorname{SEN}^{\prime}(f)(\varphi) \in T_{\Sigma^{\prime}}\right) \\
\text { iff } \delta_{\Sigma}^{\prime}(\varphi)=\epsilon_{\Sigma}^{\prime}(\varphi), \text { for all }\langle\delta, \epsilon\rangle \in \boldsymbol{\tau} .
\end{gathered}
$$

In this case, it will be said that $\tau$ defines truth in $\mathrm{M}$.

Recall that an $N$-matrix system $\left\langle\left\langle\mathrm{SEN}^{\prime},\langle F, \alpha\rangle\right\rangle, T\right\rangle$ of $d$ is Leibniz reduced if $\Omega^{N^{\prime}}(T)=\Delta^{\mathrm{SEN}^{\prime}}$ and Suszko reduced if $\tilde{\Omega}^{N^{\prime}}(T)=\Delta^{\mathrm{SEN}^{\prime}}$. Let $\mathrm{M}^{\mathrm{Su}}(\mathcal{l})$ denote the collection of all Suszko-reduced $N$-matrix systems of $\mathcal{d}$, and let $\mathrm{M}^{*}(\mathcal{d})$ denote the collection of all Leibniz-reduced $N$-matrix systems of $\ell$. Similarly, $\operatorname{LM}^{\mathrm{Su}}(\mathcal{Q})$ will denote the collection of all Suszko-reduced $N$-matrix systems of $\mathcal{d}$ of the form $\left\langle\left\langle\mathrm{SEN},\left\langle\mathrm{I}_{\mathrm{Sign}}, \iota\right\rangle\right\rangle, T\right\rangle$, and $\mathrm{LM}^{*}(\mathcal{})$ will denote the collection of all Leibniz-reduced $N$-matrix systems of $d$ of the form $\left\langle\left\langle\mathrm{SEN},\left\langle\mathrm{I}_{\text {Sign }}, \iota\right\rangle\right\rangle, T\right\rangle$. Note that 
$\left\langle\mathrm{I}_{\text {Sign }}, \iota\right\rangle:$ SEN $\rightarrow$ SEN denotes the identity $(N, N)$-epimorphic translation from SEN to SEN. (The $L$ here stands for Lindenbaum.)

It is true in general that $\mathrm{M}^{*}(\mathcal{l}) \subseteq \mathrm{M}^{\mathrm{Su}}(\mathcal{l})$, as the following lemma asserts.

Lemma 14 Let $d=\langle$ Sign, SEN, $C\rangle$ be a $\pi$-institution, with $N$ a category of natural transformations on SEN. Then $\mathrm{M}^{*}(\mathcal{l}) \subseteq \mathrm{M}^{\mathrm{Su}}(\mathcal{l})$.

Proof Let $\langle F, \alpha\rangle: \operatorname{SEN} \rightarrow \mathrm{SEN}^{\prime}$ be a surjective $\left(N, N^{\prime}\right)$-epimorphic translation, and let $T \in \operatorname{ThFam}\left(\mathcal{Q}^{\langle F, \alpha\rangle}\right)$, so that $\left\langle\left\langle\operatorname{SEN}^{\prime},\langle F, \alpha\rangle\right\rangle, T\right\rangle \in \mathrm{M}^{*}(\mathcal{Q})$. We have $\tilde{\Omega}^{N^{\prime}}(T) \leq \Omega^{N^{\prime}}(T)=\Delta^{\mathrm{SEN}^{\prime}}$. Therefore, $\left\langle\left\langle\operatorname{SEN}^{\prime},\langle F, \alpha\rangle\right\rangle, T\right\rangle \in \mathrm{M}^{\mathrm{Su}}(\mathcal{Q})$.

The following proposition characterizes implicit definability of truth in the classes $\mathrm{M}^{*}(\mathcal{l})$ and $\mathrm{M}^{\mathrm{Su}}(\mathcal{l})$ in terms of the global injectivity of the Leibniz and the Suszko operator of $\mathcal{d}$, respectively. It abstracts [23, Proposition 17].

Proposition 15 Let $d=\langle$ Sign, SEN, $C\rangle$ be a $\pi$-institution, with $N$ a category of natural transformations on SEN.

(i) Truth is implicitly definable in $\mathrm{M}^{\mathrm{Su}}(\mathcal{d})$ if and only if the Suszko operator of $d$ is globally injective.

(ii) Truth is implicitly definable in $\mathrm{M}^{*}(\mathcal{d})$ if and only if the Leibniz operator of $d$ is globally injective.

Proof We only prove (i), since (ii) may be proven similarly.

Suppose, first, that the Suszko operator of $d$ is globally injective, and let $\left\langle\left\langle\mathrm{SEN}^{\prime},\langle F, \alpha\rangle\right\rangle, T\right\rangle,\left\langle\left\langle\mathrm{SEN}^{\prime},\langle F, \alpha\rangle\right\rangle, T^{\prime}\right\rangle \in \mathrm{M}^{\mathrm{Su}}(\mathcal{Q})$. Then, we have $\tilde{\Omega}^{N^{\prime}}(T)=$ $\tilde{\Omega}^{N^{\prime}}\left(T^{\prime}\right)=\Delta^{\mathrm{SEN}^{\prime}}$, whence, by the injectivity of the Suszko operator, $T=T^{\prime}$. Hence, truth is implicitly definable in the class $\mathrm{M}^{\mathrm{Su}}(\mathcal{\ell})$.

Suppose, conversely, that truth is implicitly definable in $\mathrm{M}^{\mathrm{Su}}(\mathcal{\ell})$, and let $T, T^{\prime} \in \operatorname{ThFam}\left(\mathcal{Q}^{\langle F, \alpha\rangle}\right)$, for some surjective $\left(N, N^{\prime}\right)$-epimorphic translation $\langle F, \alpha\rangle: \mathrm{SEN} \rightarrow \mathrm{SEN}^{\prime}$, such that $\tilde{\Omega}^{N^{\prime}}(T)=\tilde{\Omega}^{N^{\prime}}\left(T^{\prime}\right)$. The two $N$-matrix systems

$$
\left\langle\left\langle\operatorname{SEN}^{\prime} / \tilde{\Omega}^{N^{\prime}}(T),\left\langle F, \pi_{F}^{\tilde{\Omega}^{N^{\prime}}(T)} \alpha\right\rangle\right\rangle, T / \tilde{\Omega}^{N^{\prime}}(T)\right\rangle
$$

and

$$
\left\langle\left\langle\operatorname{SEN}^{\prime} / \tilde{\Omega}^{N^{\prime}}\left(T^{\prime}\right),\left\langle F, \pi_{F}^{\tilde{\Omega}^{N^{\prime}}\left(T^{\prime}\right)} \alpha\right\rangle\right\rangle, T^{\prime} / \tilde{\Omega}^{N^{\prime}}\left(T^{\prime}\right)\right\rangle
$$

are in $\mathrm{M}^{\mathrm{Su}}(\mathcal{Q})$, in which, by hypothesis, truth is implicitly definable. Thus, $T=T^{\prime}$, which proves that $\tilde{\Omega}^{N^{\prime}}$ is injective.

Since $\mathrm{M}^{*}(\mathcal{l}) \subseteq \mathrm{M}^{\mathrm{Su}}(\mathcal{\ell})$, it is clearly the case that global injectivity of the Suszko operator of $d$ implies the global injectivity of the Leibniz operator of $d$, as is also the case for sentential logics (see [23, Proposition 18]).

Proposition 16 Let $d=\langle$ Sign, SEN, $C\rangle$ be a $\pi$-institution, with $N$ a category of natural transformations on SEN and $\boldsymbol{\tau}$ an $N$-translation:

(i) $\tau$ defines truth in $\mathrm{M}^{*}(\mathcal{Q})$ if and only if it defines truth in $\mathrm{M}^{\mathrm{Su}}(\mathcal{Q})$;

(ii) if $\boldsymbol{\tau}$ defines truth in $\mathrm{LM}^{*}(\mathcal{d})$, then it also defines truth in $\mathrm{LM}^{\mathrm{Su}}(\mathcal{l})$.

\section{Proof}

(i) Since $M^{*}(\mathcal{l}) \subseteq M^{S u}(\mathcal{l})$, if $\tau$ defines truth in $M^{S u}(\mathcal{l})$, then it does so also in $\mathrm{M}^{*}(\mathcal{l})$. Suppose, conversely, that $\tau$ defines truth in $\mathrm{M}^{*}(\mathcal{l})$. Let $\langle F, \alpha\rangle: \mathrm{SEN} \rightarrow \mathrm{SEN}^{\prime}$ be a surjective $\left(N, N^{\prime}\right)$-epimorphic translation, and 
let $T \in \operatorname{ThFam}\left(\mathcal{Q}^{\langle F, \alpha\rangle}\right)$, such that $\tilde{\Omega}^{N^{\prime}}(T)=\Delta^{\mathrm{SEN}^{\prime}}$, that is, such that $\left\langle\left\langle\operatorname{SEN}^{\prime},\langle F, \alpha\rangle\right\rangle, T\right\rangle \in \mathrm{M}^{\mathrm{Su}}(\mathcal{Q})$. Then we have, for all $\Sigma, \Sigma^{\prime} \in\left|\mathbf{S i g n}^{\prime}\right|$, $f \in \operatorname{Sign}^{\prime}\left(\Sigma, \Sigma^{\prime}\right)$, and all $\varphi \in \operatorname{SEN}^{\prime}(\Sigma)$,

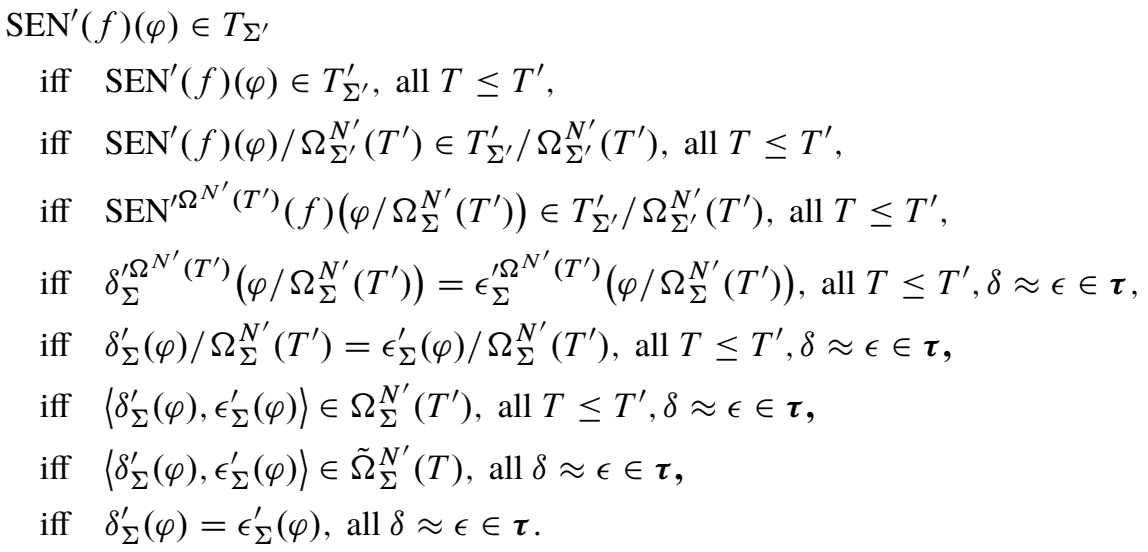

Therefore, $\boldsymbol{\tau}$ defines truth in $\mathrm{M}^{\mathrm{Su}}(\mathcal{\ell})$.

(ii) This part may proven similarly.

Let SEN : Sign $\rightarrow$ Set be a functor, with $N$ a category of natural transformations on SEN and $\tau$ an $N$-translation. Consider, also, a functor $\mathrm{SEN}^{\prime}$, with $N^{\prime}$ a category of natural transformations on $\mathrm{SEN}^{\prime}$, and an $\left(N, N^{\prime}\right)$-epimorphic translation $\langle F, \alpha\rangle: \mathrm{SEN} \rightarrow \mathrm{SEN}^{\prime}$. Denote by $T^{\boldsymbol{\tau}}$ the axiom system on $\mathrm{SEN}^{\prime}$ given, for all $\Sigma \in\left|\mathbf{S i g n}^{\prime}\right|$, by

$$
T_{\Sigma}^{\boldsymbol{\tau}}=\left\{\varphi \in \operatorname{SEN}^{\prime}(\Sigma): \delta_{\Sigma}^{\prime}(\varphi)=\epsilon_{\Sigma}^{\prime}(\varphi) \text { for all } \delta \approx \epsilon \in \boldsymbol{\tau}\right\},
$$

where by $\delta^{\prime}$ and $\epsilon^{\prime}$ are denoted the natural transformations on $\mathrm{SEN}^{\prime}$ corresponding to $\delta$ and $\epsilon$, respectively, via the $\left(N, N^{\prime}\right)$-epimorphic property. The axiom system $T^{\boldsymbol{\tau}}$ corresponds in this context to the subset

$$
F_{\mathbf{A}}^{\boldsymbol{\tau}}=\left\{a \in A: \delta_{i}^{\mathbf{A}}(a)=\epsilon_{i}^{\mathbf{A}}(a), i<n\right\}
$$

of the carrier $A$ of an algebra $\mathbf{A}$, defined via a translation $\boldsymbol{\tau}=\left\{\delta_{i}(p) \approx \epsilon_{i}(p): i<n\right\}$ in [4] (see p. 161, right before Theorem 2.3).

In the following lemma it is shown that the closure system $C^{\mathfrak{A}}$ induced by the $N$-matrix system $\mathfrak{U}=\left\langle\left\langle\mathrm{SEN}^{\prime},\langle F, \alpha\rangle\right\rangle, T^{\tau}\right\rangle$ on $\mathrm{SEN}$ is interpreted via the equations $\boldsymbol{\tau}$ into the closure system $C^{\mathcal{A}}$ on $\mathrm{SEN}^{2}$ induced by the $N$-algebraic system $\mathcal{A}=\left\langle\mathrm{SEN}^{\prime},\langle F, \alpha\rangle\right\rangle$. This will allow the formulation of an analogue of [23, Proposition 20], which was first proved as [4, Theorem 2.3]. In the categorical level, this revisits a result first proven in Voutsadakis [32].

Lemma $17 \quad$ Let $d=\langle$ Sign, SEN, $C\rangle$ be a $\pi$-institution, with $N$ a category of natural transformations on $\mathrm{SEN}, \mathcal{A}=\left\langle\mathrm{SEN}^{\prime},\langle F, \alpha\rangle\right\rangle$ an $N$-algebraic system for $\mathrm{SEN}$, and $\boldsymbol{\tau}$ an $N$-translation. Set $\mathfrak{U}=\left\langle\left\langle\mathrm{SEN}^{\prime},\langle F, \alpha\rangle\right\rangle, T^{\boldsymbol{\tau}}\right\rangle$. Then, for all $\Sigma \in|\mathbf{S i g n}|$ and all $\Phi \cup\{\varphi\} \subseteq \operatorname{SEN}(\Sigma)$,

$$
\varphi \in C_{\Sigma}^{\mathfrak{N}}(\Phi) \quad \text { iff } \quad \boldsymbol{\tau}_{\Sigma}(\varphi) \subseteq C_{\Sigma}^{\mathcal{A}}\left(\boldsymbol{\tau}_{\Sigma}(\Phi)\right) .
$$


Proof For all $\Sigma \in|\operatorname{Sign}|$ and all $\Phi \cup\{\varphi\} \subseteq \operatorname{SEN}(\Sigma)$, we have

$$
\begin{array}{cc}
\varphi \in C_{\Sigma}^{\mathfrak{N}}(\Phi) \\
\text { iff } \quad(\forall f)\left(\alpha_{\Sigma^{\prime}}(\operatorname{SEN}(f)(\Phi)) \subseteq T_{\Sigma^{\prime}}^{\boldsymbol{\tau}} \Rightarrow \alpha_{\Sigma^{\prime}}(\operatorname{SEN}(f)(\varphi)) \in T_{\Sigma^{\prime}}^{\boldsymbol{\tau}}\right) \\
\text { iff } \quad(\forall f)\left(\boldsymbol{\tau}_{F\left(\Sigma^{\prime}\right)}^{\prime}\left(\alpha_{\Sigma^{\prime}}(\operatorname{SEN}(f)(\Phi))\right) \subseteq \Delta_{F\left(\Sigma^{\prime}\right)}^{\mathrm{SEN}^{\prime}}\right. \\
\Rightarrow \boldsymbol{\tau}_{F\left(\Sigma^{\prime}\right)}^{\prime}\left(\alpha_{\Sigma^{\prime}}(\operatorname{SEN}(f)(\varphi)) \subseteq \Delta_{F\left(\Sigma^{\prime}\right)}^{\mathrm{SEN}^{\prime}}\right) \\
\text { iff } \quad(\forall f)\left(\alpha_{\Sigma^{\prime}}\left(\boldsymbol{\tau}_{\Sigma^{\prime}}(\operatorname{SEN}(f)(\Phi))\right) \subseteq \Delta_{F\left(\Sigma^{\prime}\right)}^{\mathrm{SEN}^{\prime}}\right. \\
\left.\Rightarrow \alpha_{\Sigma^{\prime}}\left(\boldsymbol{\tau}_{\Sigma^{\prime}}(\operatorname{SEN}(f)(\varphi))\right) \subseteq \Delta_{F\left(\Sigma^{\prime}\right)}^{\mathrm{SEN}^{\prime}}\right) \\
\text { iff } \quad(\forall f)\left(\alpha_{\Sigma^{\prime}}\left(\operatorname{SEN}(f)\left(\boldsymbol{\tau}_{\Sigma}(\Phi)\right)\right) \subseteq \Delta_{F\left(\Sigma^{\prime}\right)}^{\left.\mathrm{SEN}^{\prime}\right)}\right. \\
\left.\Rightarrow \alpha_{\Sigma^{\prime}}\left(\operatorname{SEN}(f)\left(\boldsymbol{\tau}_{\Sigma}(\varphi)\right)\right) \subseteq \Delta_{F\left(\Sigma^{\prime}\right)}^{\mathrm{SEN}^{\prime}}\right) \\
\text { iff } \quad \boldsymbol{\tau}_{\Sigma}(\varphi) \subseteq C_{\Sigma}^{\mathcal{A}}\left(\boldsymbol{\tau}_{\Sigma}(\Phi)\right) .
\end{array}
$$

In this proof $(\forall f)$ stands as an abbreviation for the quantifications $\left(\forall \Sigma^{\prime} \in|\operatorname{Sign}|\right)$ $\left(\forall f \in \operatorname{Sign}\left(\Sigma, \Sigma^{\prime}\right)\right)$.

Theorems 18 and 19, which follow, are direct consequences of Lemma 17. The first asserts that a $\boldsymbol{\tau}$-algebraic model of a given $\pi$-institution $d$ gives rise through the use of $T^{\boldsymbol{\tau}}$ to a matrix model of $\boldsymbol{d}$. The second concludes that, more generally, the same process allows the generation of a matrix semantics of a given $\pi$-institution from a $\boldsymbol{\tau}$-algebraic semantics along similar lines.

Theorem 18 Let $d=\langle$ Sign, SEN, $C\rangle$ be a $\pi$-institution, with $N$ a category of natural transformations on $\mathrm{SEN}, \mathcal{A}=\left\langle\mathrm{SEN}^{\prime},\langle F, \alpha\rangle\right\rangle$ an $N$-algebraic system for $\mathrm{SEN}$, and $\boldsymbol{\tau}$ an $N$-translation. Then $\mathcal{A}$ is a $\boldsymbol{\tau}$-algebraic model of $\mathcal{d}$ if and only if $\mathfrak{U}=\left\langle\left\langle\mathrm{SEN}^{\prime},\langle F, \alpha\rangle\right\rangle, T^{\boldsymbol{\tau}}\right\rangle$ is an $N$-matrix system model of $d$.

Theorem 19 Suppose that $d=\langle$ Sign, SEN, $C\rangle$ is a $\pi$-institution, with $N$ a category of natural transformations on $\mathrm{SEN}, \mathrm{F}=\left\{\left\langle\mathrm{SEN}^{i},\left\langle F^{i}, \alpha^{i}\right\rangle\right\rangle: i \in I\right\}$ a collection of $N$-algebraic systems for $\mathrm{SEN}$, and $\boldsymbol{\tau}$ an $N$-translation. Then $\mathrm{F}$ is a $\boldsymbol{\tau}$-algebraic semantics for $d$ if and only if $\mathrm{M}=\left\{\left\langle\left\langle\mathrm{SEN}^{i},\left\langle F^{i}, \alpha^{i}\right\rangle\right\rangle,\left(T^{i}\right)^{\boldsymbol{\tau}}\right\rangle: i \in I\right\}$ is an $N$-matrix system semantics for $d$.

Theorem 19 has the following corollary, which is the promised analogue of [23, Proposition 20].

Corollary $20 \quad$ Suppose that $d=\langle$ Sign, SEN, $C\rangle$ is a $\pi$-institution, with $N$ a category of natural transformations on SEN. A class of $N$-algebraic systems is an $N$-algebraic semantics for $d$ if and only if it is the class of all $N$-algebraic reducts of some $N$-matrix system semantics for $d$, in which truth is equationally definable.

Proof In fact, by Theorem 19, $\mathrm{F}=\left\{\left\langle\mathrm{SEN}^{i},\left\langle F^{i}, \alpha^{i}\right\rangle\right\rangle: i \in I\right\}$ is an $N$-algebraic system semantics iff $\mathrm{M}=\left\{\left\langle\left\langle\mathrm{SEN}^{i},\left\langle F^{i}, \alpha^{i}\right\rangle\right\rangle,\left(T^{i}\right)^{\boldsymbol{\tau}}\right\rangle: i \in I\right\}$ is an $N$-matrix system semantics for $d$ and in the latter truth is clearly equationally definable via $\boldsymbol{\tau}$.

Given a $\pi$-institution $d=\langle$ Sign, SEN, $C\rangle$, with $N$ a category of natural transformations on SEN, let us adopt the notation $\operatorname{LA}^{\mathrm{Su}}(\mathcal{l}), \mathrm{LA}^{*}(\mathcal{l})$, and $\mathrm{A}^{*}(\mathcal{l})$ to denote the classes of $N$-algebraic system reducts of $N$-matrix systems in the classes $\operatorname{LM}^{\mathrm{Su}}(\mathcal{\ell})$, $\operatorname{LM}^{*}(\mathcal{d})$, and $\mathrm{M}^{*}(\mathcal{d})$, respectively. Then, Theorem 19 yields the following. 
Corollary $21 \quad$ Let $d=\langle$ Sign, SEN, $C\rangle$ be a $\pi$-institution, with $N$ a category of natural transformations on $N$, and $\boldsymbol{\tau}$ an $N$-translation. If $\boldsymbol{\tau}$ defines truth in $\mathrm{LM}^{\mathrm{Su}}(\mathcal{Q})$, $\mathrm{LM}^{*}(\mathcal{l})$, or $\mathrm{M}^{*}(\mathcal{l})$, then $\mathrm{LA}^{\mathrm{Su}}(\mathcal{l}), \mathrm{LA}^{*}(\mathcal{l})$, or $\mathrm{A}^{*}(\mathcal{l})$, respectively, is a $\tau$-algebraic system semantics for $d$.

Raftery shows, using [23, Example 1, p. 116], that the following hold for a deductive system 8 .

(i) Equational definability of truth in $\mathrm{LMod}^{\mathrm{Su}} \delta$ does not imply the equational, or even the implicit, definability of truth in LMod* 8 .

(ii) $\operatorname{Alg}^{*} \delta$ being a $\boldsymbol{\tau}$-algebraic semantics for $\delta$ does not necessarily imply that $\boldsymbol{\tau}$ defines truth in $\operatorname{Mod}^{*} \delta$, nor even that truth is implicit definable in $\operatorname{Mod}^{*} \delta$.

(iii) The injectivity of the Suszko operator on the theories of a deductive system does not imply the existence of theorems.

Taking into account the fact that all deductive systems in the sense of [23] provide examples of $\pi$-institutions over a trivial category of signatures, Raftery's conclusions hold also for $\pi$-institutions. More precisely, given a $\pi$-institution $d=\langle$ Sign, SEN, $C\rangle$, with $N$ a category of natural transformations on SEN, and an $N$-translation $\boldsymbol{\tau}$,

- the definability of truth in the class $\operatorname{LM}^{\mathrm{Su}}(\mathcal{l})$ does not imply the equational or even implicit definability of truth in $\operatorname{LM}^{*}(\mathcal{Q})$;

- $\mathrm{A}^{*}(\mathcal{\ell})$ being a $\boldsymbol{\tau}$-algebraic system semantics for $\delta$ does not necessarily imply that $\boldsymbol{\tau}$ defines truth in $\mathrm{M}^{*}(\mathcal{\ell})$ nor even that truth is implicitly definable in $M^{*}(d)$;

- The injectivity of the Suszko operator on the theory families of $d$ does not imply existence of theorems in $\mathcal{l}$, that is, that $\operatorname{Thm}_{\Sigma}(\mathcal{l}) \neq \varnothing$, for some $\Sigma \in|\operatorname{Sign}|$.

\section{Testing for Equational Definability}

Let $d=\langle$ Sign, SEN, $C\rangle$ be a $\pi$-institution, with $N$ a category of natural transformations on $\mathrm{SEN}$, and let $\langle F, \alpha\rangle: \mathrm{SEN} \rightarrow \mathrm{SEN}^{\prime}$ be a surjective $\left(N, N^{\prime}\right)$-epimorphic translation. Define $\mathrm{M}_{\langle F, \alpha\rangle}^{*}(\mathcal{l})$ as the subclass of $\mathrm{M}^{*}(\mathcal{l})$ consisting of all Leibnizreduced $N$-matrix systems of $d$ of the form

$$
\left\langle\left\langle\operatorname{SEN}^{\prime} / \Omega^{N^{\prime}}(T),\left\langle F, \pi_{F}^{\Omega^{N^{\prime}}(T)} \alpha\right\rangle\right\rangle, T / \Omega^{N^{\prime}}(T)\right\rangle,
$$

where, of course, $T \in \operatorname{ThFam}\left(\mathcal{Q}^{\langle F, \alpha\rangle}\right)$. In particular, using this notation, we have that $\operatorname{LM}^{*}(\mathcal{l})=\mathrm{M}_{\left\langle\mathrm{ISign}_{\text {Sig }}\right\rangle}^{*}(\mathcal{l})$ and $\mathrm{M}^{*}(\mathcal{l})$ is the union of all $\mathrm{M}_{\langle F, \alpha\rangle}^{*}(\mathcal{l})$, where $\langle F, \alpha\rangle$ ranges over all surjective $\left(N, N^{\prime}\right)$-epimorphic translations. By the compatibility of $\Omega^{N^{\prime}}(T)$ with $T$, we get the following proposition paralleling in the present context [23, Proposition 22].

Proposition 22 Suppose that $d=\langle$ Sign, SEN, $C\rangle$ is a $\pi$-institution, with $N$ a category of natural transformations on $\mathrm{SEN},\langle F, \alpha\rangle: \mathrm{SEN} \rightarrow \mathrm{SEN}^{\prime}$ a surjective $\left(N, N^{\prime}\right)$-epimorphic translation, and $\boldsymbol{\tau}$ an $N$-translation. Then $\boldsymbol{\tau}$ defines truth in $\mathrm{M}_{\langle F, \alpha\rangle}^{*}(\mathcal{\ell})$ iff, for all $T \in \operatorname{ThFam}\left(\mathcal{Q}^{\langle F, \alpha\rangle}\right)$, all $\Sigma \in|\operatorname{Sign}|$, and all $\varphi \in \operatorname{SEN}(\Sigma)$,

$$
\begin{aligned}
& \left(\forall f \in \operatorname{Sign}\left(\Sigma, \Sigma^{\prime}\right)\right)\left(\alpha_{\Sigma^{\prime}}(\operatorname{SEN}(f)(\varphi)) \in T_{F\left(\Sigma^{\prime}\right)}\right) \\
& \text { iff } \boldsymbol{\tau}_{F(\Sigma)}^{\prime}\left(\alpha_{\Sigma}(\varphi)\right) \subseteq \Omega_{F(\Sigma)}^{N^{\prime}}(T) .
\end{aligned}
$$


Thus, $\boldsymbol{\tau}$ defines truth in $\mathrm{M}^{*}(\mathcal{Q})$ if and only if equivalence (6) holds for every surjective $\left(N, N^{\prime}\right)$-epimorphic translation $\langle F, \alpha\rangle: \mathrm{SEN} \rightarrow \mathrm{SEN}^{\prime}$.

Proof Suppose, first, that $\tau$ defines truth in $\mathrm{M}_{\langle F, \alpha\rangle}^{*}$, and let $\Sigma \in \mid$ Sign $\mid$ and $\varphi \in \operatorname{SEN}(\Sigma)$. We have $\tau_{F(\Sigma)}^{\prime}\left(\alpha_{\Sigma}(\varphi)\right) \subseteq \Omega_{F(\Sigma)}^{N^{\prime}}(T)$ iff, for all $\delta \approx \epsilon \in \tau$,

$$
\delta_{F(\Sigma)}^{\prime}\left(\alpha_{\Sigma}(\varphi)\right) / \Omega_{F(\Sigma)}^{N^{\prime}}(T)=\epsilon_{F(\Sigma)}^{\prime}\left(\alpha_{\Sigma}(\varphi)\right) / \Omega_{F(\Sigma)}^{N^{\prime}}(T) .
$$

But, clearly, $\left\langle\left\langle\operatorname{SEN}^{\prime \Omega^{N^{\prime}}(T)},\left\langle F, \pi_{F}^{\Omega^{N^{\prime}}(T)} \alpha\right\rangle\right\rangle, T / \Omega^{N^{\prime}}(T)\right\rangle \in \mathrm{M}_{\langle F, \alpha\rangle}^{*}(\mathcal{Q})$, whence, by hypothesis, the latter condition is equivalent to

$$
\operatorname{SEN}^{\prime \Omega^{N^{\prime}}(T)}\left(f^{\prime}\right)\left(\alpha_{\Sigma}(\varphi) / \Omega_{F(\Sigma)}^{N^{\prime}}(T)\right) \in T_{\Sigma^{\prime \prime}} / \Omega_{\Sigma^{\prime \prime}}^{N^{\prime}}(T),
$$

for all $f^{\prime} \in \operatorname{Sign}^{\prime}\left(F(\Sigma), \Sigma^{\prime \prime}\right)$. This means that, for all $f^{\prime} \in \operatorname{Sign}^{\prime}\left(F(\Sigma), \Sigma^{\prime \prime}\right)$, we have that $\operatorname{SEN}^{\prime}\left(f^{\prime}\right)\left(\alpha_{\Sigma}(\varphi)\right) / \Omega_{\Sigma^{\prime \prime}}^{N^{\prime}}(T) \in T_{\Sigma^{\prime \prime}} / \Omega_{\Sigma^{\prime \prime}}^{N^{\prime}}(T)$. By compatibility of the $N^{\prime}$-Leibniz congruence system $\Omega^{N^{\prime}}(T)$ with $T$, this condition is equivalent to $\operatorname{SEN}^{\prime}\left(f^{\prime}\right)\left(\alpha_{\Sigma}(\varphi)\right) \in T_{\Sigma^{\prime \prime}}$, for all $f^{\prime} \in \operatorname{Sign}^{\prime}\left(F(\Sigma), \Sigma^{\prime \prime}\right)$. By surjectivity of $\langle F, \alpha\rangle$ and the fact that $\langle F, \alpha\rangle$ is a translation (whence $\alpha$ is a natural transformation), we finally get the equivalence of the last condition with, for all $f \in \operatorname{Sign}\left(\Sigma, \Sigma^{\prime}\right)$, $\alpha_{\Sigma^{\prime}}\left(\operatorname{SEN}^{\prime}(f)(\varphi)\right) \in T_{F\left(\Sigma^{\prime}\right)}$. This chain of equivalences proves that condition (6) holds.

Suppose, conversely, that equivalence (6) holds, and let $\left\langle\left\langle\operatorname{SEN}^{\prime} / \Omega^{N^{\prime}}(T)\right.\right.$, $\left.\left.\left\langle F, \pi_{F}^{\Omega^{N^{\prime}}(T)} \alpha\right\rangle\right\rangle, T / \Omega^{N^{\prime}}(T)\right\rangle \in \mathrm{M}_{\langle F, \alpha\rangle}^{*}(\mathcal{d})$. Suppose, for all $f^{\prime} \in \operatorname{Sign}^{\prime}(F(\Sigma)$, $\left.\Sigma^{\prime \prime}\right)$, that we have $\operatorname{SEN}^{\prime \Omega^{N^{\prime}}(T)}\left(f^{\prime}\right)\left(\alpha_{\Sigma}(\varphi) / \Omega_{F(\Sigma)}^{N^{\prime}}(T)\right) \in T_{\Sigma^{\prime \prime}} / \Omega_{\Sigma^{\prime \prime}}^{N^{\prime}}(T)$. This is equivalent to the condition that, for all $\Sigma^{\prime} \in|\operatorname{Sign}|$ and all $f \in \operatorname{Sign}\left(\Sigma, \Sigma^{\prime}\right)$, $\operatorname{SEN}^{\prime}(F(f))\left(\alpha_{\Sigma}(\varphi)\right) / \Omega_{F\left(\Sigma^{\prime}\right)}^{N^{\prime}}(T) \in T_{F\left(\Sigma^{\prime}\right)} / \Omega_{F\left(\Sigma^{\prime}\right)}^{N^{\prime}}(T)$. By compatibility of $\Omega^{N^{\prime}}(T)$ with $T$, the latter condition is equivalent to $\operatorname{SEN}^{\prime}(F(f))\left(\alpha_{\Sigma}(\varphi)\right) \in T_{F\left(\Sigma^{\prime}\right)}$, for all $f \in \operatorname{Sign}\left(\Sigma, \Sigma^{\prime}\right)$. Now equivalence (6) may be applied to get $\boldsymbol{\tau}_{F(\Sigma)}^{\prime}\left(\alpha_{\Sigma}(\varphi)\right) \subseteq$ $\Omega_{F(\Sigma)}^{N^{\prime}}(T)$, which is equivalent to

$$
\delta_{F(\Sigma)}^{\prime \Omega^{N^{\prime}}(T)}\left(\alpha_{\Sigma}(\varphi) / \Omega_{F(\Sigma)}^{N^{\prime}}(T)\right)=\epsilon_{F(\Sigma)}^{\prime \Omega^{N^{\prime}}(T)}\left(\alpha_{\Sigma}(\varphi) / \Omega_{F(\Sigma)}^{N^{\prime}}(T)\right) .
$$

This concludes the proof that $\tau$ defines truth in $\mathrm{M}_{\langle F, \alpha\rangle}^{*}(\mathcal{l})$.

Our next goal is to establish a lemma to the effect that definability of truth has some preservation properties when it comes to composing epimorphic translations. To pave the way for Lemma 24, which is an analogue of [23, Lemma 23], we have to first prove a technical lemma, Lemma 23 , to the effect, roughly speaking, that pushing a theory family forward through such a morphism also results in a valid theory family and to establish a correspondence between the Leibniz congruence systems of these two theory families.

Lemma 23 Let $d=\langle$ Sign, SEN, $C\rangle$ be a $\pi$-institution, with $N$ a category of natural transformations on $\mathrm{SEN}$, let $\langle F, \alpha\rangle: \mathrm{SEN} \rightarrow \mathrm{SEN}^{\prime}$ be a surjective $\left(N, N^{\prime}\right)$-epimorphic translation, and let $T \in \operatorname{ThFam}\left(\mathcal{Q}^{\langle F, \alpha\rangle}\right)$. Let, also, $\langle G, \beta\rangle: \mathrm{SEN}^{\prime} \rightarrow \mathrm{SEN}^{\prime \prime}$ be a surjective $\left(N^{\prime}, N^{\prime \prime}\right)$-epimorphic translation, with $G$ an isomorphism, such that $\operatorname{Ker}(\langle G, \beta\rangle)$ is compatible with T. Then

(i) $\beta(T):=\left\{\beta_{\Sigma}\left(T_{\Sigma}\right)\right\}_{\Sigma \in\left|\operatorname{Sign}^{\prime}\right|} \in \operatorname{ThFam}\left(\mathcal{Q}^{\left\langle G F, \beta_{F} \alpha\right\rangle}\right)$, and

(ii) $\beta_{F(\Sigma)}\left(\Omega_{F(\Sigma)}^{N^{\prime}}(T)\right)=\Omega_{G(F(\Sigma))}^{N^{\prime \prime}}(\beta(T))$, for all $\Sigma \in \mid$ Sign $\mid$. 


\section{Proof}

(i) Suppose that $\Sigma \in|\mathbf{S i g n}|$ and $\Phi \cup\{\varphi\} \subseteq \operatorname{SEN}(\Sigma)$, such that $\varphi \in C_{\Sigma}(\Phi)$. Since, by hypothesis, $T \in \operatorname{ThFam}\left(\mathcal{d}^{\langle F, \alpha\rangle}\right)$, we get, taking into account Proposition 1 , that, for all $f \in \operatorname{Sign}\left(\Sigma, \Sigma^{\prime}\right)$,

$$
\alpha_{\Sigma^{\prime}}(\operatorname{SEN}(f)(\Phi)) \subseteq T_{F\left(\Sigma^{\prime}\right)} \quad \text { implies } \quad \alpha_{\Sigma^{\prime}}(\operatorname{SEN}(f)(\varphi)) \in T_{F\left(\Sigma^{\prime}\right)}
$$

Thus, if $\beta_{F\left(\Sigma^{\prime}\right)}\left(\alpha_{\Sigma^{\prime}}(\operatorname{SEN}(f)(\Phi))\right) \subseteq \beta_{F\left(\Sigma^{\prime}\right)}\left(T_{F\left(\Sigma^{\prime}\right)}\right)$, we get, by the postulated compatibility of $\operatorname{Ker}(\langle G, \beta\rangle)$ with $T$, that $\alpha_{\Sigma^{\prime}}(\operatorname{SEN}(f)(\Phi)) \subseteq T_{F\left(\Sigma^{\prime}\right)}$, whence, by (7), $\alpha_{\Sigma^{\prime}}(\operatorname{SEN}(f)(\varphi)) \in T_{F\left(\Sigma^{\prime}\right)}$ and, therefore,

$$
\beta_{F\left(\Sigma^{\prime}\right)}\left(\alpha_{\Sigma^{\prime}}(\operatorname{SEN}(f)(\varphi))\right) \in \beta_{F\left(\Sigma^{\prime}\right)}\left(T_{F\left(\Sigma^{\prime}\right)}\right) .
$$

This, again using Proposition 1 , shows that $\beta(T) \in \operatorname{ThFam}\left(\mathcal{Q}^{\langle G F, \beta \alpha\rangle}\right)$.

(ii) Let us show that $\Omega_{F(\Sigma)}^{N^{\prime}}(T)=\beta_{F(\Sigma)}^{-1}\left(\Omega_{G(F(\Sigma))}^{N^{\prime \prime}}(\beta(T))\right)$, for all $\Sigma \in$ $\mid$ Sign $\mid$. For all $\varphi, \psi \in \operatorname{SEN}^{\prime}(F(\Sigma)),\langle\varphi, \psi\rangle \in \beta_{F(\Sigma)}^{-1}\left(\Omega_{G(F(\Sigma))}^{N^{\prime \prime}}(\beta(T))\right)$ iff $\left\langle\beta_{F(\Sigma)}(\varphi), \beta_{F(\Sigma)}(\psi)\right\rangle \in \Omega_{G(F(\Sigma))}^{N^{\prime \prime}}(\beta(T))$, which is equivalent to, for all $f \in \operatorname{Sign}\left(\Sigma, \Sigma^{\prime}\right), \sigma$ in $N, \vec{\chi}^{\prime \prime} \in \operatorname{SEN}^{\prime \prime}\left(G\left(F\left(\Sigma^{\prime}\right)\right)\right)$,

$$
\begin{aligned}
& \sigma_{G\left(F\left(\Sigma^{\prime}\right)\right)}^{\prime \prime}\left(\operatorname{SEN}^{\prime \prime}(G(F(f)))\left(\beta_{F(\Sigma)}(\varphi)\right), \vec{\chi}^{\prime \prime}\right) \in \beta_{F\left(\Sigma^{\prime}\right)}\left(T_{F\left(\Sigma^{\prime}\right)}\right) \\
& \quad \text { iff } \quad \sigma_{G\left(F\left(\Sigma^{\prime}\right)\right)}^{\prime \prime}\left(\operatorname{SEN}^{\prime \prime}(G(F(f)))\left(\beta_{F(\Sigma)}(\psi)\right), \vec{\chi}^{\prime \prime}\right) \in \beta_{F\left(\Sigma^{\prime}\right)}\left(T_{F\left(\Sigma^{\prime}\right)}\right) .
\end{aligned}
$$

Equivalently, for all $f \in \operatorname{Sign}\left(\Sigma, \Sigma^{\prime}\right), \sigma$ in $N, \vec{\chi}^{\prime} \in \operatorname{SEN}^{\prime}\left(F\left(\Sigma^{\prime}\right)\right)$,

$$
\begin{aligned}
& \sigma_{G\left(F\left(\Sigma^{\prime}\right)\right)}^{\prime \prime}\left(\beta_{F\left(\Sigma^{\prime}\right)}\left(\operatorname{SEN}^{\prime}(F(f))(\varphi)\right), \beta_{F\left(\Sigma^{\prime}\right)}\left(\vec{\chi}^{\prime}\right)\right) \in \beta_{F\left(\Sigma^{\prime}\right)}\left(T_{F\left(\Sigma^{\prime}\right)}\right) \\
& \quad \text { iff } \quad \sigma_{G\left(F\left(\Sigma^{\prime}\right)\right)}^{\prime \prime}\left(\beta_{F\left(\Sigma^{\prime}\right)}\left(\operatorname{SEN}^{\prime}(F(f))(\psi)\right), \beta_{F\left(\Sigma^{\prime}\right)}\left(\vec{\chi}^{\prime}\right)\right) \in \beta_{F\left(\Sigma^{\prime}\right)}\left(T_{F\left(\Sigma^{\prime}\right)}\right) .
\end{aligned}
$$

This holds, iff, for all $f \in \operatorname{Sign}\left(\Sigma, \Sigma^{\prime}\right), \sigma$ in $N, \vec{\chi}^{\prime} \in \operatorname{SEN}^{\prime}\left(F\left(\Sigma^{\prime}\right)\right)$,

$$
\begin{aligned}
& \beta_{F\left(\Sigma^{\prime}\right)}\left(\sigma_{F\left(\Sigma^{\prime}\right)}^{\prime}\left(\operatorname{SEN}^{\prime}(F(f))(\varphi)\right), \vec{\chi}^{\prime}\right) \in \beta_{F\left(\Sigma^{\prime}\right)}\left(T_{F\left(\Sigma^{\prime}\right)}\right) \\
& \quad \text { iff } \beta_{F\left(\Sigma^{\prime}\right)}\left(\sigma_{F\left(\Sigma^{\prime}\right)}^{\prime}\left(\operatorname{SEN}^{\prime}(F(f))(\psi)\right), \vec{\chi}^{\prime}\right) \in \beta_{F\left(\Sigma^{\prime}\right)}\left(T_{F\left(\Sigma^{\prime}\right)}\right),
\end{aligned}
$$

which, taking into account the postulated compatibility of $\operatorname{Ker}(\langle G, \beta\rangle)$ with $T$, is, in turn, equivalent to, for all $f \in \operatorname{Sign}\left(\Sigma, \Sigma^{\prime}\right), \sigma$ in $N$, $\vec{\chi}^{\prime} \in \operatorname{SEN}^{\prime}\left(F\left(\Sigma^{\prime}\right)\right)$,

$$
\begin{aligned}
& \left.\sigma_{F\left(\Sigma^{\prime}\right)}^{\prime}\left(\operatorname{SEN}^{\prime}(F(f))(\varphi)\right), \vec{\chi}^{\prime}\right) \in T_{F\left(\Sigma^{\prime}\right)} \\
& \left.\quad \text { iff } \quad \sigma_{F\left(\Sigma^{\prime}\right)}^{\prime}\left(\operatorname{SEN}^{\prime}(F(f))(\psi)\right), \vec{\chi}^{\prime}\right) \in T_{F\left(\Sigma^{\prime}\right)},
\end{aligned}
$$

that is, to $\langle\varphi, \psi\rangle \in \Omega_{F(\Sigma)}^{N^{\prime}}(T)$.

In the next lemma we study the effect of applying surjective translations in both the forward and the backward directions to the definability of truth. It turns out that the definability of truth is always preserved in the forward direction but that one has to impose the additional conditions introduced in Lemma 23 to ensure preservation of definability when applying an epimorphic translation in the backward direction. These two results are expressed in parts (1) and (2) of the following lemma, respectively. The lemma forms an analogue of [23, Lemma 23] in the categorical framework. 
Lemma 24 Let $d=\langle$ Sign, SEN, $C\rangle$ be a $\pi$-institution, with $N$ a category of natural transformations on SEN. Let, also, $\langle F, \alpha\rangle: \mathrm{SEN} \rightarrow \mathrm{SEN}^{\prime}$ be a surjective $\left(N, N^{\prime}\right)$-epimorphic translation, and let $\langle G, \beta\rangle: \mathrm{SEN}^{\prime} \rightarrow \mathrm{SEN}^{\prime \prime}$ be a surjective $\left(N^{\prime}, N^{\prime \prime}\right)$-epimorphic translation.

(1) If an $N$-translation $\tau$ defines truth in $\mathrm{M}_{\langle F, \alpha\rangle}^{*}(\mathcal{Q})$, then it also defines truth in $\mathrm{M}_{\left\langle G F, \beta_{F} \alpha\right\rangle}^{*}(\mathcal{d})$.

(2) Conversely, if an $N$-translation $\tau$ defines truth in $\mathrm{M}_{\left\langle G F, \beta_{F} \alpha\right\rangle}^{*}(\mathcal{d}), G$ is an isomorphism, and $\operatorname{Ker}(\langle G, \beta\rangle)$ is compatible with all theory families $T \in \operatorname{ThFam}\left(\mathcal{Q}^{\langle F, \alpha\rangle}\right)$, then $\tau$ also defines truth in $\mathrm{M}_{\langle F, \alpha\rangle}^{*}(\mathcal{\ell})$.

Proof Assume that, for all $T \in \operatorname{ThFam}\left(\mathcal{L}^{\langle F, \alpha\rangle}\right)$, all $\Sigma \in|\mathbf{S i g n}|$, and all $\varphi \in \operatorname{SEN}(\Sigma), \alpha_{\Sigma^{\prime}}(\operatorname{SEN}(f)(\varphi)) \in T_{F\left(\Sigma^{\prime}\right)}$, for all $f \in \operatorname{Sign}\left(\Sigma, \Sigma^{\prime}\right)$, if and only if $\boldsymbol{\tau}_{F(\Sigma)}^{\prime}\left(\alpha_{\Sigma}(\varphi)\right) \subseteq \Omega_{F(\Sigma)}^{N^{\prime}}(T)$. Let $T^{\prime} \in \operatorname{ThFam}\left(\mathcal{d}^{\left\langle G F, \beta_{F} \alpha\right\rangle}\right), \Sigma \in \mid$ Sign $\mid$, and $\varphi \in \operatorname{SEN}(\Sigma)$. Then

$$
\begin{aligned}
\left(\forall f \in \operatorname{Sign}\left(\Sigma, \Sigma^{\prime}\right)\right)\left(\beta_{F\left(\Sigma^{\prime}\right)}\left(\alpha_{\Sigma^{\prime}}(\operatorname{SEN}(f)(\varphi))\right) \in T_{G\left(F\left(\Sigma^{\prime}\right)\right)}^{\prime}\right) \\
\text { iff } \quad\left(\forall f \in \operatorname{Sign}\left(\Sigma, \Sigma^{\prime}\right)\right)\left(\alpha_{\Sigma^{\prime}}(\operatorname{SEN}(f)(\varphi)) \in \beta_{F\left(\Sigma^{\prime}\right)}^{-1}\left(T_{G\left(F\left(\Sigma^{\prime}\right)\right)}^{\prime}\right)\right) \\
\text { iff } \quad \boldsymbol{\tau}_{F(\Sigma)}^{\prime}\left(\alpha_{\Sigma}(\varphi)\right) \subseteq \Omega_{F(\Sigma)}^{N^{\prime}}\left(\beta^{-1}\left(T^{\prime}\right)\right) \\
\text { iff } \quad \boldsymbol{\tau}_{F(\Sigma)}^{\prime}\left(\alpha_{\Sigma}(\varphi)\right) \subseteq \beta_{F(\Sigma)}^{-1}\left(\Omega_{G(F(\Sigma))}^{N^{\prime \prime}}\left(T^{\prime}\right)\right) \\
\text { iff } \quad \beta_{F(\Sigma)}\left(\boldsymbol{\tau}_{F(\Sigma)}^{\prime}\left(\alpha_{\Sigma}(\varphi)\right)\right) \subseteq \Omega_{G(F(\Sigma))}^{N^{\prime \prime}}\left(T^{\prime}\right) \\
\text { iff } \quad \boldsymbol{\tau}_{G(F(\Sigma))}^{\prime \prime}\left(\beta_{F(\Sigma)}\left(\alpha_{\Sigma}(\varphi)\right)\right) \subseteq \Omega_{G(F(\Sigma))}^{N^{\prime \prime}}\left(T^{\prime}\right) .
\end{aligned}
$$

Thus, $\boldsymbol{\tau}$ also defines truth in $\mathrm{M}_{\left\langle G F, \beta_{F} \alpha\right\rangle}^{*}(\mathcal{l})$.

Assume, conversely, that, for all $T^{\prime} \in \operatorname{ThFam}\left(\mathcal{d}^{\left\langle G F, \beta_{F} \alpha\right\rangle}\right)$, all $\Sigma \in|\mathbf{S i g n}|$, and all $\varphi \in \operatorname{SEN}(\Sigma), \beta_{F\left(\Sigma^{\prime}\right)}\left(\alpha_{\Sigma^{\prime}}(\operatorname{SEN}(f)(\varphi))\right) \in T_{G\left(F\left(\Sigma^{\prime}\right)\right)}^{\prime}$, for all $f \in \operatorname{Sign}\left(\Sigma, \Sigma^{\prime}\right)$, if and only if $\boldsymbol{\tau}_{G(F(\Sigma))}^{\prime \prime}\left(\beta_{F(\Sigma)}\left(\alpha_{\Sigma}(\varphi)\right)\right) \subseteq \Omega_{G(F(\Sigma))}^{N^{\prime \prime}}\left(T^{\prime}\right)$, that $G$ is an isomorphism, and that $\operatorname{Ker}(\langle G, \beta\rangle)$ is compatible with every $T \in \operatorname{ThFam}\left(\mathcal{\alpha}^{\langle F, \alpha\rangle}\right)$. Recall that, if $T \in \operatorname{ThFam}\left(\mathcal{Q}^{\langle F, \alpha\rangle}\right)$, then, by Lemma $23, \beta(T) \in \operatorname{ThFam}\left(\mathcal{Q}^{\left\langle G F, \beta_{F} \alpha\right\rangle}\right)$. Hence, for all $\Sigma \in|\operatorname{Sign}|$ and all $\varphi \in \operatorname{SEN}(\Sigma)$, we have

$$
\begin{aligned}
\left(\forall f \in \operatorname{Sign}\left(\Sigma, \Sigma^{\prime}\right)\right)\left(\alpha_{\Sigma^{\prime}}(\operatorname{SEN}(f)(\varphi)) \in T_{F\left(\Sigma^{\prime}\right)}\right) \\
\quad \text { iff } \quad\left(\forall f \in \operatorname{Sign}\left(\Sigma, \Sigma^{\prime}\right)\right)\left(\beta_{F\left(\Sigma^{\prime}\right)}\left(\alpha_{\Sigma^{\prime}}(\operatorname{SEN}(f)(\varphi))\right) \in \beta_{F\left(\Sigma^{\prime}\right)}\left(T_{F\left(\Sigma^{\prime}\right)}\right)\right) \\
\text { iff } \quad \boldsymbol{\tau}_{G(F(\Sigma))}^{\prime \prime}\left(\beta_{F(\Sigma)}\left(\alpha_{\Sigma}(\varphi)\right)\right) \subseteq \Omega_{G(F(\Sigma))}^{N^{\prime}}(\beta(T)) \\
\text { iff } \quad \beta_{F(\Sigma)}\left(\boldsymbol{\tau}_{F(\Sigma)}^{\prime}\left(\alpha_{\Sigma}(\varphi)\right)\right) \subseteq \beta_{F(\Sigma)}\left(\Omega_{F(\Sigma)}^{N^{\prime}}(T)\right) \\
\text { iff } \quad \boldsymbol{\tau}_{F(\Sigma)}^{\prime}\left(\alpha_{\Sigma}(\varphi)\right) \subseteq \Omega_{F(\Sigma)}^{N^{\prime}}(T) .
\end{aligned}
$$

In the last chain of equivalences we have used both part (2) of Lemma 23 and the compatibility of $\operatorname{Ker}(\langle G, \beta\rangle)$ with $T \in \operatorname{ThFam}\left(\mathcal{Q}^{\langle F, \alpha\rangle}\right)$. The chain shows that $\boldsymbol{\tau}$ defines truth on $\mathrm{M}_{\langle F, \alpha\rangle}^{*}(\mathcal{l})$.

The following result asserts that definability of truth for the class of Leibniz-reduced matrix system models of a $\pi$-institution is equivalent to definability of truth for the subclass of Leibniz-reduced Lindenbaum matrix system models of $\mathcal{~}$. Moreover, this property is characterized by another condition concerning definability of truth 
in Leibniz-reduced matrix system models on subclasses of the algebraic reducts of Leibniz-reduced matrix system models that form appropriate algebraic semantics.

Theorem $25 \quad$ Let $d=\langle$ Sign, SEN, $C\rangle$ be a $\pi$-institution, with $N$ a category of natural transformations on SEN and $\boldsymbol{\tau}$ an $N$-translation. Then, the following are equivalent:

(i) $\tau$ defines truth in $\mathrm{M}^{*}(\mathcal{Q})$;

(ii) $\tau$ defines truth in $\mathrm{LM}^{*}(\mathcal{d})$;

(iii) $\mathrm{LA}^{*}(\mathcal{l})$ is a $\boldsymbol{\tau}$-algebraic system semantics for $d$ and, for every subclass $\mathrm{K}$ of $N$-algebraic systems in $\mathrm{A}^{*}(\mathcal{d})$, that is a $\tau$-algebraic system semantics for $d$, $\tau$ defines truth in $\bigcup_{\left\langle\operatorname{SEN}^{\prime},\langle F, \alpha\rangle\right\rangle \in \mathrm{K}} \mathrm{M}_{\langle F, \alpha\rangle}^{*}(\mathcal{\ell})$.

\section{Proof}

(i) $\rightarrow$ (ii) Since $\operatorname{LM}^{*}(\mathcal{d}) \subseteq \mathrm{M}^{*}(\mathcal{d})$, this implication is trivial.

(ii) $\rightarrow$ (iii) Since $\tau$ defines truth in $\operatorname{LM}^{*}(\mathcal{l})$, by Corollary 21 , the class $\operatorname{LA}^{*}(\mathcal{l})$ is a $\tau$-algebraic system semantics for $\mathcal{\ell}$. Hence, there exists at least one subclass of $A^{*}(\mathcal{\ell})$ that is a $\boldsymbol{\tau}$-algebraic system semantics for $\mathcal{\ell}$. Let $\mathrm{K}$ be such a subclass. Consider an $N$-algebraic system $\left\langle\mathrm{SEN}^{\prime},\langle F, \alpha\rangle\right\rangle \in \mathrm{K}$. Since $\tau$ defines truth in $\operatorname{LM}^{*}(\mathcal{l})$ and $\langle F, \alpha\rangle$ is surjective, by Lemma 24(1), $\tau$ also defines truth in $\mathrm{M}_{\langle F, \alpha\rangle}^{*}(\mathcal{\ell})$.

(iii) $\rightarrow$ (i) The hypothesis implies that $\tau$ defines truth in $\operatorname{LM}^{*}(\mathcal{l})$. Let $\langle F, \alpha\rangle$ : $\mathrm{SEN} \rightarrow \mathrm{SEN}^{\prime}$ be a surjective $\left(N, N^{\prime}\right)$-epimorphic translation. Consider the kernel $N$-congruence system $\theta:=\operatorname{Ker}(\langle F, \alpha\rangle)$ on SEN together with the associated natural projection $\left(N, N^{\theta}\right)$-epimorphic translation $\left\langle\mathrm{I}_{\text {Sign }}, \pi^{\theta}\right\rangle:$ SEN $\rightarrow$ SEN $/ \theta$ (see [33]). Then, there exists an $\left(N^{\theta}, N^{\prime}\right)$-epimorphic translation $\left\langle F, \alpha^{*}\right\rangle: \mathrm{SEN} / \theta \rightarrow \mathrm{SEN}^{\prime}$ that makes the following triangle commute:

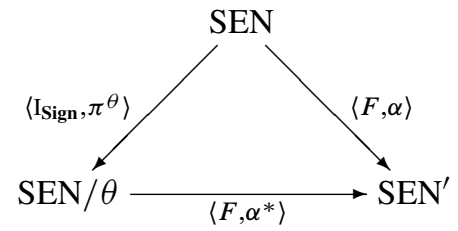

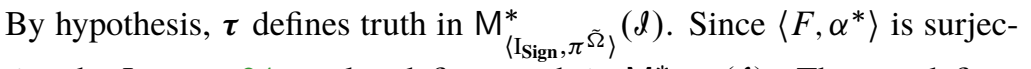
tive, by Lemma 24, $\boldsymbol{\tau}$ also defines truth in $\mathrm{M}_{\langle F, \alpha\rangle}^{*}(\mathcal{l})$. Thus, $\boldsymbol{\tau}$ defines truth in $\mathrm{M}^{*}(\mathcal{l})=\bigcup_{\langle F, \alpha\rangle} \mathrm{M}_{\langle F, \alpha\rangle}^{*}(\mathcal{l})$.

Proposition 16 and Theorem 25 have the following consequence.

Corollary $26 \quad$ Let $d=\langle\operatorname{Sign}, \mathrm{SEN}, C\rangle$ be a $\pi$-institution, with $N$ a category of natural transformations on SEN and $\boldsymbol{\tau}$ an $N$-translation. If $\boldsymbol{\tau}$ defines truth in $\operatorname{LM}^{*}(\mathcal{l})$, then each of $\mathrm{LA}^{*}(\mathcal{\ell}), \mathrm{A}^{*}(\mathcal{\ell})$, and $\mathrm{A}^{\mathrm{Su}}(\mathcal{l})$ is a $\boldsymbol{\tau}$-algebraic system semantics for $d$.

\section{Truth-Equational $\pi$-Institutions}

A $\pi$-institution $d=\langle$ Sign, SEN, $C\rangle$, with $N$ a category of natural transformations on SEN, will be called $N$-truth-equational if truth is equationally definable by an 
$N$-translation in the class $\operatorname{LM}^{*}(\mathcal{\ell})$. Any $N$-translation $\tau$ that defines truth in $\mathrm{LM}^{*}(\mathcal{\ell})$ is said to witness the $N$-truth-equationality of $d$.

By Proposition 16, if $d$ is $N$-truth-equational, then truth is also equationally definable in $\mathrm{M}^{\mathrm{Su}}(\mathcal{l})$ and, hence, also in $\mathrm{M}^{*}(\mathcal{l})$ and $\mathrm{LM}^{\mathrm{Su}}(\mathcal{l})$. In contrast, Raftery shows in [23, Example 1] that, for a deductive system $\delta$, equational definability of truth in $\mathrm{LMod}^{\mathrm{Su}} \&$ does not imply equational definability of truth in any of the other matrix semantics for $\delta$. His result carries over to the context of $\pi$-institutions to the effect that equational definability of truth in $\operatorname{LM}^{\mathrm{Su}}(\mathcal{l})$ does not entail equational definability of truth in any of the other matrix system model classes.

Theorem 27 of [23] asserts the truth-equationality of a deductive system $\delta$ whose Leibniz operator is completely order reflecting on the lattice of theories of $\delta$. We attempt to provide a generalization of this result in the categorical level. Unfortunately, we are forced to impose a rather stringent condition on the $\pi$-institution under consideration in order to establish such an analogue. We show that it holds for what we call $N$-Suszko term $\pi$-institutions, a subclass of the class of term $\pi$-institutions considered in [25]. It is conjectured that this result does not hold in general for arbitrary $\pi$-institutions.

Recall from [25] (see Voutsadakis [34] and also, for generalizations, Gil-Férez [15], Galatos and Tsinakis [14], and, more recently, Galatos and Gil-Férez [13]) that, given a category Sign and a sentence functor SEN : Sign $\rightarrow$ Set, SEN is said to be term if there exists $V \in|\mathbf{S i g n}|$ and $v \in \operatorname{SEN}(V)$, such that

- for all $\Sigma \in|\operatorname{Sign}|$ and all $\varphi \in \operatorname{SEN}(\Sigma)$, there exists $f_{\langle\Sigma, \varphi\rangle}: V \rightarrow \Sigma$, such that $\operatorname{SEN}\left(f_{\langle\Sigma, \varphi\rangle}\right)(v)=\varphi$, and

- for all $\Sigma^{\prime} \in|\operatorname{Sign}|$ and all $f \in \operatorname{Sign}\left(\Sigma, \Sigma^{\prime}\right), f \circ f_{\langle\Sigma, \varphi\rangle}=f_{\left\langle\Sigma^{\prime}, \operatorname{SEN}(f)(\varphi)\right\rangle}$, for all $\varphi \in \operatorname{SEN}(\Sigma)$.

The pair $\langle V, v\rangle$ is called a source signature-variable pair. A $\pi$-institution $d=$ $\langle$ Sign, SEN, $C\rangle$, with SEN a term sentence functor is called a term $\pi$-institution.

Assume, next that $d=\langle$ Sign, SEN, $C\rangle$ is a term $\pi$-institution, with $N$ a category of natural transformations on SEN. Let $Z=\operatorname{Thm}^{[\langle V, v\rangle]}=\left\{\operatorname{Thm}_{\Sigma}^{[\langle V, v\rangle]}\right\}_{\Sigma \in \mid \text { Sign } \mid}$, with

$$
\operatorname{Thm}_{\Sigma}^{[\langle V, v\rangle]}= \begin{cases}C_{V}(v) & \text { if } \Sigma=V, \\ C_{\Sigma}(\emptyset) & \text { if } \Sigma \neq V .\end{cases}
$$

Consider the Suszko $N$-congruence system $\tilde{\Omega}^{N}(Z)$, and define $\tau:$ SEN $\rightarrow \mathrm{SEN}^{2}$ by setting, for all $\Sigma \in|\operatorname{Sign}|$ and all $\varphi \in \operatorname{SEN}(\Sigma)$,

$$
\tau_{\Sigma}(\varphi)=\operatorname{SEN}^{2}\left(f_{\langle\Sigma, \varphi\rangle}\right)\left(\tilde{\Omega}_{V}^{N}(Z)\right) .
$$

It is not difficult to see that $\tau: \mathrm{SEN} \rightarrow \mathrm{SEN}^{2}$ is a natural transformation. We have, for all $\Sigma, \Sigma^{\prime} \in|\operatorname{Sign}|$, all $f \in \operatorname{Sign}\left(\Sigma, \Sigma^{\prime}\right)$, and all $\varphi \in \operatorname{SEN}(\Sigma)$,

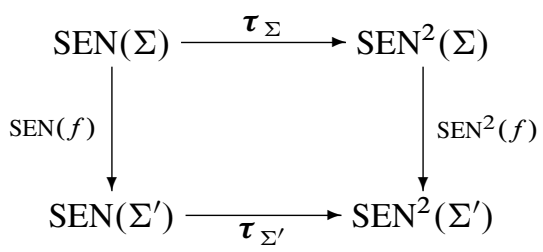




$$
\begin{aligned}
\operatorname{SEN}^{2}(f)\left(\tau_{\Sigma}(\varphi)\right) & =\operatorname{SEN}^{2}(f)\left(\operatorname{SEN}^{2}\left(f_{\langle\Sigma, \varphi\rangle}\right)^{2}\left(\tilde{\Omega}_{V}^{N}(Z)\right)\right) \\
& =\operatorname{SEN}^{2}\left(f_{\left\langle\Sigma^{\prime}, \operatorname{SEN}(f)(\varphi)\right\rangle}\right)\left(\tilde{\Omega}_{V}^{N}(Z)\right) \\
& =\boldsymbol{\tau}_{\Sigma^{\prime}}(\operatorname{SEN}(f)(\varphi)) .
\end{aligned}
$$

Therefore, there exists a set of pairs $\langle\delta, \epsilon\rangle$ of natural transformations $\delta^{i}, \epsilon^{i}$ : SEN $\rightarrow$ SEN, $i \in I$, such that $\tau=\left\{\left\langle\delta^{i}, \epsilon^{i}\right\rangle: i \in I\right\}$. The natural transformations $\delta^{i}, \epsilon^{i}:$ SEN $\rightarrow$ SEN may not necessarily be in $N$. Nor is it necessarily the case that, for all $\Sigma, \Sigma^{\prime} \in|\operatorname{Sign}|$, all $\varphi \in \operatorname{SEN}(\Sigma)$, and all $f \in \operatorname{Sign}\left(\Sigma, \Sigma^{\prime}\right)$,

$$
\tilde{\Omega}_{\Sigma^{\prime}}^{N}\left(\operatorname{Thm}^{\langle\Sigma, \varphi\rangle}\right)=\operatorname{SEN}^{2}\left(f_{\left\langle\Sigma^{\prime}, \operatorname{SEN}(f)(\varphi)\right\rangle}\right)\left(\tilde{\Omega}_{V}^{N}(Z)\right),
$$

where $\operatorname{Thm}^{\langle\Sigma, \varphi\rangle}$ denotes the theory system of $d$ generated by $\langle\Sigma, \varphi\rangle$ as in [29, Section 3]. If these two conditions hold for the $\pi$-institution $d=\langle$ Sign, SEN, $C\rangle$, for some source signature-variable pair $\langle V, v\rangle$, we call $d$ an $N$-Suszko term $\pi$-institution (with respect to the source signature-variable pair $\langle V, v\rangle$ ). In other words, a term $\pi$-institution $d=\langle$ Sign, $\operatorname{SEN}, C\rangle$, with a source signature-variable pair $\langle V, v\rangle$ and with $N$ a category of natural transformations on SEN, is $N$-Suszko term with respect to $\langle V, v\rangle$ if, for all $\Sigma \in|\operatorname{Sign}|$ and all $\varphi \in \operatorname{SEN}(\Sigma)$,

1. the natural transformation $\tau$, defined, starting from $\langle V, v\rangle$, by

$$
\tau_{\Sigma}(\varphi)=\operatorname{SEN}^{2}\left(f_{\langle\Sigma, \varphi\rangle}\right)\left(\tilde{\Omega}_{V}^{N}(Z)\right)
$$

consists of a set of pairs of unary natural transformations in $N$; and

2. $\tilde{\Omega}_{\Sigma^{\prime}}^{N}\left(\operatorname{Thm}^{\langle\Sigma, \varphi\rangle}\right)=\operatorname{SEN}^{2}\left(f_{\left\langle\Sigma^{\prime}, \operatorname{SEN}(f)(\varphi)\right\rangle}\right)\left(\tilde{\Omega}_{V}^{N}(Z)\right)=\tau_{\Sigma^{\prime}}(\operatorname{SEN}(f)(\varphi))$, for all $\Sigma^{\prime} \in|\operatorname{Sign}|$ and all $f \in \operatorname{Sign}\left(\Sigma, \Sigma^{\prime}\right)$.

For $N$-Suszko term $\pi$-institutions, we are able to prove the following theorem, which is an analogue of one of the main theorems, [23, Theorem 27]. It provides a key ingredient in the characterization of the property of truth-equationality of an $N$-Suszko term $\pi$-institution in terms of the complete order reflexivity of the Leibniz operator on the theory families of the $\pi$-institution.

Theorem 27 Let Sign be a category, let SEN : Sign $\rightarrow$ Set be a term sentence functor, with source signature-variable pair $\langle V, v\rangle$, and let $N$ be a category of natural transformations on $\mathrm{SEN}$. If $d=\langle$ Sign, $\mathrm{SEN}, C\rangle$ is $N$-Suszko term with respect to $\langle V, v\rangle$ and the $N$-Leibniz operator of $d$ is completely order reflecting on $\operatorname{ThFam}(\mathcal{d})$, then $d$ is $N$-truth-equational.

Proof For all $\Sigma \in|\operatorname{Sign}|$ and all $\varphi \in \operatorname{SEN}(\Sigma)$, let, as before,

$$
\tau_{\Sigma}(\varphi)=\operatorname{SEN}^{2}\left(f_{\langle\Sigma, \varphi\rangle}\right)\left(\tilde{\Omega}_{V}^{N}(Z)\right),
$$

where $Z=\operatorname{Thm}^{[\langle V, v\rangle]}$. Since $d$ is $N$-Suszko term with respect to $\langle V, v\rangle, \boldsymbol{\tau}$ is an $N$-translation. It suffices to show that $\boldsymbol{\tau}$ defines truth in $\operatorname{LM}^{*}(\mathcal{l})$. By Proposition 22, it suffices to show that, for all $T \in \operatorname{ThFam}(\mathcal{Q})$, all $\Sigma \in|\operatorname{Sign}|$, and all $\varphi \in \operatorname{SEN}(\Sigma)$,

$$
\left(\forall f \in \operatorname{Sign}\left(\Sigma, \Sigma^{\prime}\right)\right)\left(\operatorname{SEN}(f)(\varphi) \in T_{\Sigma^{\prime}}\right) \quad \text { iff } \quad \tau_{\Sigma}(\varphi) \subseteq \Omega_{\Sigma}^{N}(T) .
$$

For the implication from left to right, we have, for all $\Sigma \in|\operatorname{Sign}|$ and all $\varphi \in$ $\operatorname{SEN}(\Sigma)$, such that $\operatorname{SEN}(f)(\varphi) \in T_{\Sigma^{\prime}}$, for all $\Sigma^{\prime} \in|\operatorname{Sign}|$ and all $f \in \operatorname{Sign}\left(\Sigma, \Sigma^{\prime}\right)$,

$$
\begin{aligned}
\tau_{\Sigma}(\varphi) & =\operatorname{SEN}^{2}\left(f_{\langle\Sigma, \varphi\rangle}\right)\left(\tilde{\Omega}_{V}^{N}(Z)\right) \\
& =\tilde{\Omega}_{\Sigma}^{N}\left(\operatorname{Thm}^{\langle\Sigma, \varphi\rangle}\right) \quad(\text { since } d \text { is } N \text {-Suszko term })
\end{aligned}
$$




$$
\begin{aligned}
& \subseteq \tilde{\Omega}_{\Sigma}^{N}(T) \text { (by the monotonicity of the } N \text {-Suszko operator) } \\
& \subseteq \Omega_{\Sigma}^{N}(T) .
\end{aligned}
$$

For the reverse implication, assume that $T \in \operatorname{ThFam}(\mathcal{\ell}), \Sigma \in|\operatorname{Sign}|$, and $\varphi \in \operatorname{SEN}(\Sigma)$, such that $\boldsymbol{\tau}_{\Sigma}(\varphi) \subseteq \Omega_{\Sigma}^{N}(T)$. Then, for all $\Sigma^{\prime} \in|\operatorname{Sign}|$ and all $f \in \operatorname{Sign}\left(\Sigma, \Sigma^{\prime}\right)$, we have

$$
\begin{aligned}
\tilde{\Omega}_{\Sigma^{\prime}}^{N}\left(\operatorname{Thm}^{\langle\Sigma, \varphi\rangle}\right) & =\tau_{\Sigma^{\prime}}(\operatorname{SEN}(f)(\varphi)) \quad(\text { since } d \text { is } N \text {-Suszko term }) \\
& =\operatorname{SEN}^{2}(f)\left(\tau_{\Sigma}(\varphi)\right) \\
& \subseteq \operatorname{SEN}^{2}(f)\left(\Omega_{\Sigma}^{N}(T)\right) \\
& \subseteq \Omega_{\Sigma^{\prime}}^{N}(T) .
\end{aligned}
$$

Since this holds for all $\Sigma^{\prime} \in \mid$ Sign $\mid$, we have that $\tilde{\Omega}^{N}\left(\operatorname{Thm}^{\langle\Sigma, \varphi\rangle}\right) \leq \Omega^{N}(T)$. Thus, by the complete order reflexivity of the $N$-Leibniz operator of $d$, we get that $\operatorname{Thm}^{\langle\Sigma, \varphi\rangle} \leq T$ and, therefore, $\operatorname{SEN}(f)(\varphi) \in T_{\Sigma^{\prime}}$, for all $\Sigma^{\prime} \in|\operatorname{Sign}|$ and all $f \in \operatorname{Sign}\left(\Sigma, \Sigma^{\prime}\right)$, as was to be shown.

Theorem $28 \quad$ Let $d=\langle$ Sign, SEN, $C\rangle$ be a $\pi$-institution, and let $N$ be a category of natural transformations on SEN. Consider the following conditions:

(i) $d$ is $N$-truth-equational;

(ii) truth is $N$-equationally definable in $\mathrm{M}^{\mathrm{Su}}(\mathcal{d})$;

(iii) the $N$-Suszko operator of $d$ is globally injective;

(iv) for every surjective $\left(N, N^{\prime}\right)$-epimorphic translation $\langle F, \alpha\rangle: \mathrm{SEN} \rightarrow \mathrm{SEN}^{\prime}$ and every theory family $T \in \operatorname{ThFam}\left(\mathcal{Q}^{\langle F, \alpha\rangle}\right)$, the least theory family of $\mathcal{\alpha}^{\left\langle F, \pi_{F}^{\tilde{\Omega}^{N^{\prime}}(T)} \alpha\right\rangle}$ is $T / \tilde{\Omega}^{N^{\prime}}(T)$;

(v) the $N$-Leibniz operator of $d$ is globally completely order reflecting;

(vi) the $N$-Leibniz operator of $d$ is completely order reflecting on the collection $\operatorname{ThFam}(\mathcal{d})$.

We have, in general, that $(i) \leftrightarrow($ ii $) \rightarrow($ iii $) \leftrightarrow(i v) \leftrightarrow(v) \rightarrow(v i)$. Moreover, if $d$ is $N$-Suszko term, then all six conditions are equivalent.

Proof We have that (i) $\leftrightarrow$ (ii) holds by Theorem 25 and Proposition 16. The implication (ii) $\rightarrow$ (iii) follows from Proposition 15. The equivalence (iii) $\leftrightarrow$ (iv) is the content of Theorem 9, whereas the equivalence (iii) $\leftrightarrow$ (v) is the content of Theorem 13 . The implication $(v) \rightarrow$ (vi) is trivial. Theorem 27 gives the implication (vi) $\rightarrow$ (i) under the additional hypothesis that $d$ is $N$-Suszko term.

It is worth noting that Raftery in [23, Example 2] furnishes a finitary deductive system with an elementary class of Leibniz-reduced matrix models, having an algebraic semantics with respect to a finite translation, whose Leibniz operator is globally injective but which is not truth-equational. Thus, global injectivity of the Leibniz operator together with possessing an algebraic semantics does not guarantee truthequationality. Moreover, in [23, Example 3], it is shown that global injectivity of the Leibniz operator does not entail the existence of an algebraic semantics even for deductive systems that are finitary and possess an elementary class of Leibniz-reduced matrix models. 


\section{References}

[1] Barr, M., and C. Wells, Category Theory for Computing Science, Prentice-Hall International Series in Computer Science, Prentice-Hall, Upper Saddle River, N. J., 1990; available online at http://www.math.mcgill.ca/triples/Barr-Wells-ctcs.pdf (accessed 30 November 2014). 354

[2] Blok, W. J., and D. Pigozzi, "Protoalgebraic logics," Studia Logica, vol. 45 (1986), pp. 337-69. Zbl 0622.03020. MR 0884144. DOI 10.1007/BF00370269. 352

[3] Blok, W. J., and D. Pigozzi, "Algebraizable logics," Memoirs of the American Mathematical Society, vol. 77 (1989), no. 396. MR 0973361. DOI 10.1090/memo/0396. 352, $353,354,358,359$

[4] Blok, W. J., and J. Rebagliato, "Algebraic semantics for deductive systems," Studia Logica, vol. 74 (2003), pp. 153-80. Zbl 1049.03008. MR 1996595. DOI 10.1023/A:1024626023417. 353, 366

[5] Borceux, F., Handbook of Categorical Algebra, Vol. I, Encyclopedia of Mathematics and Its Applications, Cambridge Univ. Press, Cambridge, 1994. 354

[6] Czelakowski, J., "Equivalential logics, I," Studia Logica, vol. 40 (1981), pp. 227-36. Zbl 0476.03032. MR 0665717. DOI 10.1007/BF02584057. 352

[7] Czelakowski, J., "Equivalential logics, II,” Studia Logica, vol. 40 (1981), pp. 355-72. Zbl 0492.03008. MR 0692902. DOI 10.1007/BF00401654. 352

[8] Czelakowski, J., Protoalgebraic Logics, vol. 10 of Trends in Logic-Studia Logica Library, Kluwer, Dordrecht, 2001. MR 1828895. DOI 10.1007/978-94-017-2807-2. 352, 359

[9] Czelakowski, J., “The Suszko operator, Part I," pp. 181-231 in Abstract Algebraic Logic, Part II (Barcelona, 1997), vol. 74 of Studia Logica, Springer, Dordrecht, 2003. MR 1996596. DOI 10.1023/A:1024678007488. 352, 358, 359, 361

[10] Fiadeiro, J., and A. Sernadas, "Structuring theories on consequence," pp. 44-72 in Recent Trends in Data Type Specification (Gullane, Scotland, 1987), edited by D. Sannella and A. Tarlecki, vol. 332 of Lecture Notes in Computer Science, Springer, Berlin, 1988. 354

[11] Font, J. M., and R. Jansana, A General Algebraic Semantics for Sentential Logics, vol. 7 of Lecture Notes in Logic, Springer, Berlin, 1996. MR 1421569. DOI 10.1007/978-3-662-21591-3. 352, 355, 359

[12] Font, J. M., R. Jansana, and D. Pigozzi, "A survey of abstract algebraic logic," Studia Logica, vol. 74 (2003), pp. 13-97. Zbl 1057.03058. MR 1996593. DOI 10.1023/A:1024621922509. 352, 353, 359

[13] Galatos, N., and J. Gil-Férez, "Modules over quantaloids: Applications to the isomorphism problem in algebraic logic and $\pi$-institutions," preprint, to appear in Journal of Pure and Applied Algebra. 373

[14] Galatos, N., and C. Tsinakis, "Equivalence of closure operators: An order-theoretic and categorical perspective," Journal of Symbolic Logic, vol. 74 (2009), pp. 780-810. MR 2548478. DOI 10.2178/jsl/1245158085. 373

[15] Gil-Férez, J., "Multi-term $\pi$-institutions and their equivalence," Mathematical Logic Quarterly, vol. 52 (2006), pp. 505-26. Zbl 1111.03056. MR 2268702. DOI 10.1002/malq.200610010. 373

[16] Goguen, J. A., and R. M. Burstall, "Introducing institutions," pp. 221-56 in Logics of Programs (Pittsburgh, 1983), edited by E. Clarke and D. Kozen, vol. 164 of Lecture Notes in Computer Science, Springer, Berlin, 1984. MR 0778942. DOI 10.1007/3-540-12896-4_366. 354

[17] Goguen, J. A., and R. M. Burstall, "Institutions: Abstract model theory for specification and programming," Journal of the Association for Computing Machinery, vol. 39 (1992), pp. 95-146. Zbl 0799.68134. MR 1147298. DOI 10.1145/147508.147524. 354 
[18] Herrmann, B., "Equivalential and algebraizable logics," Studia Logica, vol. 57 (1996), pp. 419-36. Zbl 0864.03043. MR 1416744. DOI 10.1007/BF00370843. 352

[19] Herrmann, B., "Characterizing equivalential and algebraizable logics by the Leibniz operator," Studia Logica, vol. 58 (1997), pp. 305-23. MR 1439022. DOI 10.1023/A:1004979825733. 352

[20] Herrmann, B., "Equivalential logics and definability of truth," Ph.D. dissertation, Freie Universitat Berlin, Berlin, 1993. 352

[21] Mac Lane, S., Categories for the Working Mathematician, vol. 5 of Graduate Texts in Mathematics, Springer, New York, 1971. MR 0354798. 354

[22] Prucnal, T., and A. Wronski, "An algebraic characterization of the notion of structural completeness," Polish Academy of Sciences Institute of Philosophy and Sociology Bulletin of the Section of Logic, vol. 3 (1974), pp. 30-33. MR 0403950. 352

[23] Raftery, J. G., "The equational definability of truth predicates," Reports on Mathematical Logic, vol. 41 (2006), pp. 95-149. Zbl 1136.03011. MR 2376004. 353, 354, 358, 360, $361,362,363,365,366,367,368,369,370,373,374,375$

[24] Voutsadakis, G., "Categorical abstract algebraic logic: Algebraizable institutions," Applied Categorical Structures, vol. 10 (2002), pp. 531-68. Zbl 1028.03051. MR 1941391. DOI 10.1023/A:1020990419514. 354

[25] Voutsadakis, G., "Categorical abstract algebraic logic: Equivalent institutions," pp. 275-311 in Abstract Algebraic Logic, Part II (Barcelona, 1997), vol. 74 of Studia Logica, Springer, Dordrecht, 2003. Zbl 1043.03049. MR 1996598. DOI 10.1023/A:1024682108396. 354, 373

[26] Voutsadakis, G., "Categorical abstract algebraic logic: $(\mathcal{Q}, N)$-algebraic systems," $A p$ plied Categorical Structures, vol. 13 (2005), pp. 265-80. Zbl 1089.03057. MR 2167794. DOI 10.1007/s10485-005-5797-5. 355

[27] Voutsadakis, G., "Categorical abstract algebraic logic: Models of $\pi$-institutions," Notre Dame Journal of Formal Logic, vol. 46 (2005), pp. 439-60. Zbl 1089.03058. MR 2183054. DOI 10.1305/ndjfl/1134397662. 355, 356

[28] Voutsadakis, G., "Categorical abstract algebraic logic: More on protoalgebraicity," Notre Dame Journal of Formal Logic, vol. 47 (2006), pp. 487-514. Zbl 1134.03044. MR 2272084. DOI 10.1305/ndjfl/1168352663. 358

[29] Voutsadakis, G., "Categorical abstract algebraic logic: Prealgebraicity and protoalgebraicity," Studia Logica, vol. 85 (2007), pp. 217-51. MR 2329535. DOI 10.1007/s11225-007-9029-x. 358, 359, 360, 374

[30] Voutsadakis, G., "Categorical abstract algebraic logic: Strong version of a protoalgebraic pi-institution," Reports on Mathematical Logic, vol. 42 (2007), pp. 19-46. Zbl 1133.03044. MR 2378219. 356

[31] Voutsadakis, G., "Categorical abstract algebraic logic: The categorical Suszko operator," Mathematical Logic Quarterly, vol. 53 (2007), pp. 616-35. Zbl 1134.03045. MR 2351584. DOI 10.1002/malq.200710020. 358, 359

[32] Voutsadakis, G., "Categorical abstract algebraic logic: Algebraic semantics for $\pi$-institutions," Mathematical Logic Quarterly, vol. 59 (2013), pp. 177-200. MR 3066738. DOI 10.1002/malq.200920103. 361, 366

[33] Voutsadakis, G., "Categorical abstract algebraic logic: Tarski congruence systems, logical morphisms and logical quotients," Ph.D. dissertation, Iowa State University, Ames, 1998. MR 2697808. 359, 372

[34] Voutsadakis, G., "Categorical abstract algebraic logic: Equivalence of closure systems," preprint, http://www.voutsadakis.com/RESEARCH/papers.html (accessed 30 November 2014). 373 
School of Mathematics and Computer Science Lake Superior State University

650 W. Easterday Avenue

Sault Sainte Marie, Michigan 49783

USA

gvoutsad@Issu.edu

http://www.voutsadakis.com/main.html 